UdeM-GPP-TH-09-183

IFIBA-TH-09-001

\title{
Vertex Displacements for Acausal Particles: Testing the Lee-Wick Standard Model at the LHC
}

\author{
Ezequiel Álvarez, ${ }^{1}$ Leandro Da Rold, ${ }^{2}$ Carlos Schat, ${ }^{1,3}$ and Alejandro Szynkman ${ }^{4}$ \\ ${ }^{1}$ CONICET, IFIBA and Departamento de Física, FCEyN, \\ Universidad de Buenos Aires, Ciudad Universitaria, \\ Pab.1, (1428) Buenos Aires, Argentina \\ ${ }^{2}$ CONICET and Centro Atómico Bariloche, \\ Av. Bustillo 9500, 8400, S. C. de Bariloche, Argentina \\ ${ }^{3}$ Department of Physics and Astronomy, \\ Ohio University, Athens, Ohio 45701, USA \\ ${ }^{4}$ Physique des Particules, Université de Montréal, C.P. 6128, \\ succ. centre-ville, Montréal, QC, Canada H3C $3 J^{\text {ry }}$
}

(Dated: October 14, 2009)

\begin{abstract}
We propose to search for wrong displaced vertices, where decay products of the secondary vertex move towards the primary vertex instead of away from it, as a signature for microscopic violation of causality. We analyze in detail the leptonic sector of the recently proposed Lee-Wick Standard Model, which provides a well motivated framework to study acausal effects. We find that, assuming Minimal Flavor Violation, the Lee-Wick partners of the electron, $\tilde{\ell}^{e}$ and $\tilde{e}$, can produce measurable wrong vertices at the LHC, the most promising channel being $q \bar{q} \longrightarrow \overline{\tilde{\ell}} \tilde{\ell}^{e} \longrightarrow e^{+} e^{-} j j j j$. A MonteCarlo simulation using MadGraph/MadEvent suggests that for $M_{\ell} \lesssim 450 \mathrm{GeV}$ the measurement of these acausal vertex displacements should be accessible in the LHC era.
\end{abstract}




\section{INTRODUCTION}

Despite all the phenomenological success of the Standard Model (SM), the large quantum corrections to the Higgs potential require a fine tuning that makes it unnatural as a complete theory. This fine tuning is known as the hierarchy problem and can be avoided if there is new physics at the $\mathrm{TeV}$ scale, the energy scale that will be explored at the Large Hadron Collider (LHC). Finding a solution to the hierarchy problem that can also be tested soon by the upcoming experiments at the LHC provides a very strong motivation for building extensions to the Standard Model.

A recent proposal that solves the hierarchy problem is the Lee-Wick Standard Model (LWSM) [1], based on ideas of Lee and Wick [2, 3] for a finite theory of quantum electrodynamics. In the LWSM each SM particle has a Lee-Wick (LW) partner of the same statistics. The only new parameters of the model are the LW mass matrices. LW particles have a kinetic term with the opposite sign to the usual one for SM particles, leading to partial cancellations in loop corrections that eliminate quadratic divergences.

Different theoretical and phenomenological aspects of the LWSM have been discussed recently in Refs. [4, 5, 6, 17, 8, 9, 10, 11, 12, 13, 14, 15, 16, 17, 18, 19, 20].

A very interesting and distinctive feature of the LWSM is its acausal behavior. This might be a serious problem of the theory if acausal effects could persist to macroscopic scales, leading to paradoxes. For a general discussion of causality see Coleman lectures, Ref. [21]. It has been argued that if LW particles decay fast enough the violation of causality would happen on a very small time scale and macroscopic causality would still be preserved as an emergent property [19].

Besides its phenomenological interest, LW-type theories also give the theoretical framework to discuss acausality. A LW version of the $\mathrm{O}(\mathrm{N})$ model has been used to check the consistency of the acausal theory to all orders in perturbation theory [19]. The authors have shown that there is a well-defined S-matrix in scattering processes, that provides a one-to-one map from the past to the future. Although a similar result for the LWSM would be much more difficult to obtain, the result of Ref. [19] is encouraging. The properties in thermal equilibrium have also been examined to further check if multiple scattering can lead to macroscopic acausal behavior [20].

The main question we address here is the following. Given a theory that allows for 
microscopic violations of causality, but which is still free from paradoxes at the macroscopic level: Is it possible to propose an observable that could reveal a microscopic violation of causality by solely analyzing the in and out states of the S-matrix? We answer this question in the affirmative and to this purpose we define a wrong vertex displacement as a vertex displacement in which the decay products coming from the secondary vertex have a total momentum that points from the secondary to the primary vertex and its invariant mass corresponds to a new resonance ${ }^{1}$. As we discuss in the next Section, acausal theories give rise to wrong vertex displacements.

As a direct application of the general question here addressed and the above defined observable, we analyze in this article the possibility of measuring a wrong vertex displacement as a signature of a LW resonance at the LHC. We investigate which LW particles could have the smallest widths to produce the largest wrong vertex displacements.

To answer this question we study in detail the flavor interactions of the leptonic sector in the LWSM. We obtain that if the LW sector satisfies Minimal Flavor Violation (MFV), the best candidate to produce wrong displaced vertices is the LW electron associated to the SU(2) doublet. In order to investigate the possibility of measuring a wrong vertex displacement at the LHC, we perform Monte Carlo simulations using MadGraph/MadEvent [23] to study which are the conditions such that there is a LW particle stable enough to produce an observable wrong vertex displacement. We find that for LW masses satisfying $M_{\ell} \lesssim 450 \mathrm{GeV}$, these particles would produce displacements in the transverse plane greater than $20 \mu \mathrm{m}$ at cross-sections that would be measurable in the LHC era. Another good candidate to produce observable wrong vertex displacements is the LW partner of the electron in the singlet representation of the gauge group. Other LW particles seem to be out of the reach of the LHC for these purposes, but could still be observed by direct production, although it would be more difficult to discriminate them from other candidates of new physics. A wrong displaced vertex on the other hand, would be a characteristic signature for an acausal particle.

The paper is organized as follows. In Section III we show that acausal resonances lead to wrong vertex displacements and argue that, in the LWSM, LW leptons are the best

\footnotetext{
${ }^{1}$ A similar experimental signature, but without requiring the total momentum of the decay products to have a fixed invariant mass, is called "large negative impact parameter (LNIP)" in Ref. 22].
} 
candidates to observe them. In Section III we analyze the flavor structure in the leptonic sector of the LWSM, and we obtain the mass eigenstates and the interactions in this basis. In Section IV we compute the widths for different LW-leptonic physical states and conclude that the first generation of LW-charged leptons will have a considerably smaller width than other LW particles. In Section $\nabla$ we discuss which are the necessary conditions to identify the desired events and we design a set of cuts and procedures to isolate our signal at the LHC. We end with our conclusions in Section VI. Details of the diagonalization of the leptonic mass matrix and the interactions between mass eigenstates can be found in the Appendices.

\section{WRONG VERTEX DISPLACEMENTS}

One of the main purposes of this article is to propose the measurement of wrong vertex displacement associated to processes that go through LW resonances at the LHC. A normal (non-LW) resonant state, produced by some initial particle collision, propagates typically for the space of time allowed by its mean lifetime before it decays into lighter particles. Unlike the usual case, the opposite occurs for LW resonances: decay products precede production. However, for the theory to be consistent it should forbid the temporal exploration of this time-scale. On the other hand, it is possible to probe spatially this acausal behavior by means of the detection of displaced decay vertices corresponding to relatively 'long-lived' LW resonances and through the measurement of certain kinetic variables related to the decay products.

When a normal resonance is created with non-zero momentum, the total momentum of the decay products points from the decay point (secondary vertex), in the outgoing direction from where the resonance was created (primary vertex) ${ }^{2}$. Of course, this is a direct consequence of the outgoing direction of the momentum of the resonance.

On the other hand, the presence of LW - or any acausal- resonances may be detected by observing an opposite pattern: the resulting momentum of the decay products heads

\footnotetext{
${ }^{2}$ A familiar case where this has been observed corresponds to the $B$ mesons. After these particles are created, they travel some distance in the laboratory frame before they decay. One can infer the time elapsed between the production and decay points to obtain a measure of the $B$ mesons mean lifetimes. This kind of measurements have been performed by using different techniques at LEP, Tevatron and the $B$ factories 24$]$.
} 
inwards, from the secondary towards the primary vertex.

This particular behavior may be understood by means of a quantum mechanical argument as well as in a formal way using an S-matrix description. In the first case, Lee and Wick [2, 3] and Coleman [21] have argued that, in a process going through a LW resonance in the center of mass frame, decay products would appear before collision takes place. In this picture, incoming particles collide in the same spatial point where decay products come from. Moreover, incoming and outgoing particles have zero total momentum in this frame of reference. If this whole process is boosted in some direction and its events are accordingly Lorentz transformed, one easily retrieves the wrong displaced vertex described in the previous paragraph. (Moreover, just a Galileo transformation is enough for this purpose.) We show pictorially in Fig. 1 this production and decay process mediated by an acausal resonance in both reference frames.

Alternatively, and in a more formal way, a recent paper by Grinstein, O'Connell and Wise [19] may also help us to understand this behavior. They compute the transition amplitude between arbitrary two-particle states mediated by LW resonances using the S-matrix description of quantum field theory. The final result of this calculation, which corresponds to Eq. (46) in their paper, is the following

$$
\left\langle\psi_{\text {out }} \mid \psi_{\text {in }}\right\rangle \simeq \frac{g^{2} \sqrt{M}}{2\left(2 \pi \sqrt{\omega^{2}}\right)^{3 / 2}} \hat{F}\left(-M \omega / \sqrt{\omega^{2}}\right) \hat{G}\left(-M \omega / \sqrt{\omega^{2}}\right) e^{i M \sqrt{\omega^{2}}} e^{-\Gamma \sqrt{\omega^{2}} / 2} .
$$

The relevant argument for our analysis lies in the functions $\hat{F}$ and $\hat{G}$ which essentially contain the information about the distribution of momenta in the initial and final states, respectively. Both $\hat{F}$ and $\hat{G}$ are peaked $\operatorname{around}^{3} q \approx-M \omega / \sqrt{\omega^{2}}$, where $q$ stands for the total four-momentum of the incoming/outgoing particles, $\omega=z_{D}-z_{P}$ is the space-time separation between the positions of the decay $\left(z_{D}\right)$ and production $\left(z_{P}\right)$ vertices associated to the intermediate LW-resonant state, and $M$ represents its mass. Due to the relative sign between $q$ and $\omega$, the secondary vertex is displaced from the primary towards the opposite direction of $q$. Consider now the case where the total momentum of the incoming particles is different from zero in the laboratory reference frame, as for instance $t_{0}$ in the target frame in Fig. 1. This means that if $q$ points towards the right in the figure, then the traces of the

\footnotetext{
${ }^{3}$ Instead, the relation $q \approx M \omega / \sqrt{\omega^{2}}$ is satisfied by a causal resonances. See Eq. (34) in Ref. [19].
} 

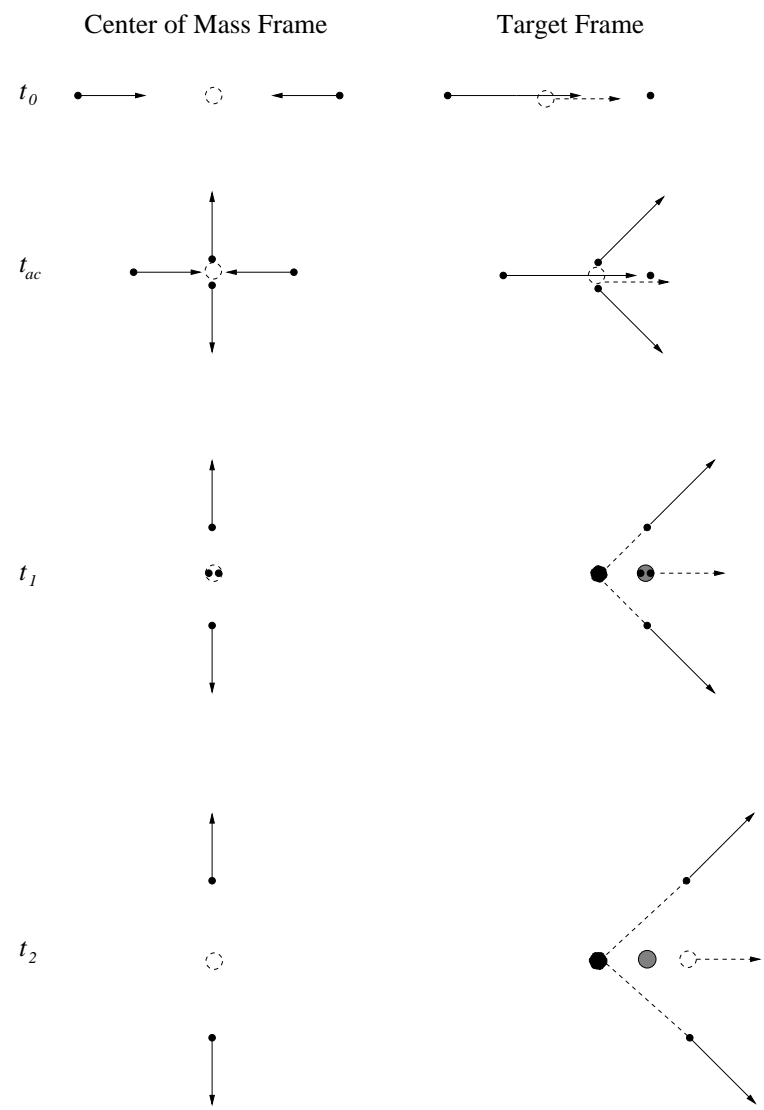

FIG. 1: Pictorial description of the collision and decay process through an intermediate LW resonance, as seen in two reference frames. The dashed circle is the center of mass of the system. The gray (black) disk signals the position of the primary (secondary) vertex in the target frame. The acausal behaviour sets in at time $t_{a c}$, when the decay products appear before the LW resonance is created. At long times before and after the collision $\left(t_{0}, t_{2}\right)$ the configuration seems equivalent to the causal case, except that the extrapolated secondary vertex lies to the left of the primary vertex and the decay products move towards the primary vertex instead of away from it. As it is explained in the text, the same final result ( $t_{2}$ as an out state of a $t_{0}$ in state) is rigorously derived using the S-matrix formalism without inquiring what happens during the process, that is, without a microscopic description between $t_{a c}$ and $t_{1}$.

decay products should appear to come from a secondary vertex located at the left of the primary vertex, as it happens at $t_{2}$ in the target frame in Fig. 1. As a matter of fact in an S-matrix description one has access only to these two times $\left(t_{0}\right.$ and $\left.t_{2}\right)$, since it is senseless to inquire what happens during the process. This is the way the atypical displacement 
pattern of secondary vertices arises in scattering processes mediated by LW resonances in the S-matrix description. Other consequences of Eq. (11) are further discussed in Ref. [19]. As a last remark, we mention that this equation has been derived in the narrow resonance approximation which is valid in the case under study here (typically, for a LW electron with $\mathrm{M} \sim 300 \mathrm{GeV}$, we find $\left.\Gamma \sim 10^{-11} \mathrm{GeV}\right)$.

There is a point we should clarify in order to avoid misunderstandings in the interpretation of what we have discussed in the previous paragraphs. Within the context of the S-matrix formalism, it does not make sense to rise the question about the time nor the position the vertices take place since we only deal with asymptotic states in this framework. In order to establish a proper connection between this theoretical approach and observations, it is necessary to perform measurements far away from the region where the resonance propagates and at times well separated from the interval of time the propagation takes. These conditions are indeed satisfied at the LHC: measurements are carried out far outside the interaction region and within a relative long-time window that contains the entire process of creation and decay of the LW resonances. Thus, the vertex positions are not measured (in the sense that measurements do not perturb the dynamics of the system at the vertices) but only indirectly obtained by extrapolating the traces left by the outgoing particles along the detectors.

Once we have justified how the LWSM (or any theory with acausal particles) could give rise to a wrong vertex displacement, we may focus on how this signature could be observed in this specific model.

The first important question to address is about the existence of general reasons to expect a LW particle to have a very small width. The width is determined by the masses and the interactions between the particles involved in the decay process. Present constrains on the LW masses coming from EWPT [15, 17, 18] indicate that the lowest allowed masses for LW quarks and LW-intermediate bosons are close to $3 \mathrm{TeV}$, whereas the Higgs sector is only constrained to have masses above $\sim 400 \mathrm{GeV}[25]$. On the the other hand, there are no limits on LW-leptonic masses other than those coming from direct lepton search, which give a lowest bound of $\sim 100 \mathrm{GeV}$ [26]. Besides the mass range difference between the LW leptons and the LW quarks, these last have the additional interaction with the gluon and the LW gluon. Therefore, the LW quarks are expected to have a total width greater than the LW leptons. A similar reasoning holds for the LW-intermediate bosons, which have stronger 
constraints and prompt decays. Hence, we conclude that LW leptons may be expected to be less unstable ${ }^{4}$ and, therefore, the more propitious to give a wrong vertex displacement signature.

The next important feature when explicitly analyzing the LWSM is that, since the creation of a single LW lepton $\tilde{\ell}^{e}$ is suppressed in this model, is more likely to observe two wrong displaced vertices than one. For instance in the process $p p \rightarrow \overline{\tilde{\ell^{e}}} \tilde{\ell}^{e} \rightarrow X_{1} X_{2}$ both $\tilde{\ell}^{e}$ 's would produce wrong vertex displacements and hence their decay products $X_{1}$ and $X_{2}$ would have their respective total momentum pointing towards the primary vertex. This situation, as expected at the LHC, is shown in Fig. 2.
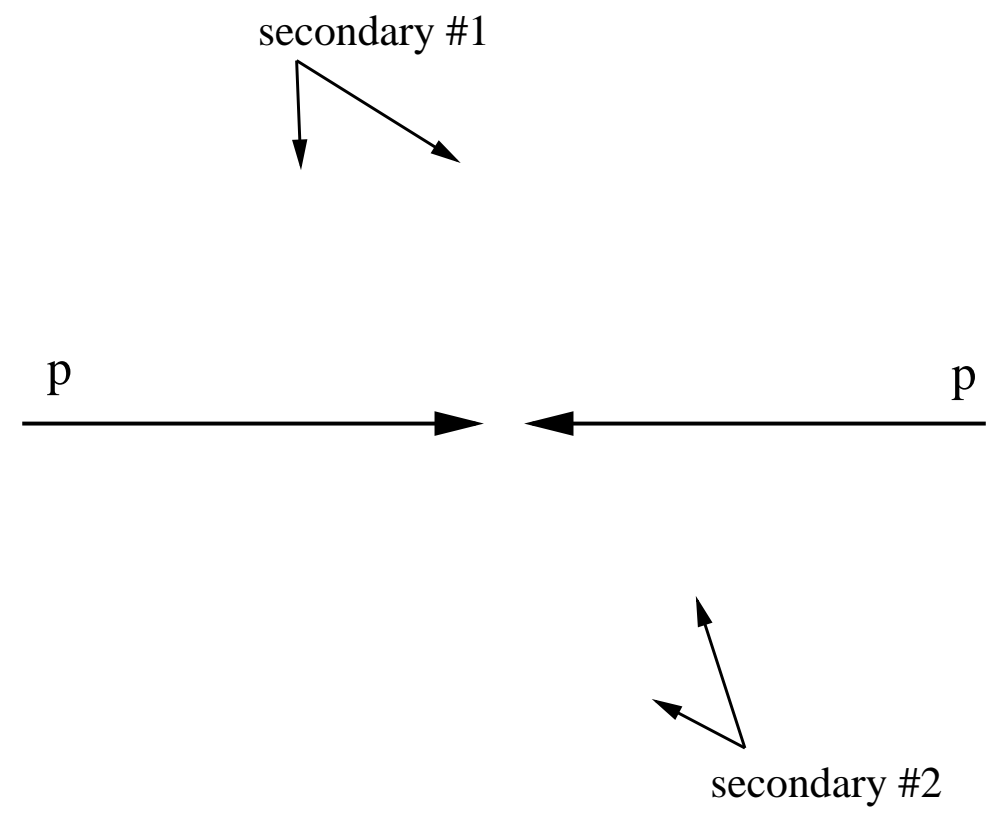

FIG. 2: Two wrong vertex displacement expected in the LWSM. Decay products coming from both secondary vertices travel towards the primary vertex, instead of moving away from it.

In the next Section we study the LW-leptonic sector in order to obtain the mass eigenstates that should be created to give the sought signal, and their interactions.

\footnotetext{
${ }^{4}$ A few days before the submission of this work, Ref. [25] reported a low bound for the Higgs mass in $\sim 400$ $\mathrm{GeV}$. Although we have not performed the due analysis, we expect that the many decay channels open for the LW Higgs would increase its width considerably above the one expected for the LW leptons. In any case, we clarify that this does not modify our results.
} 


\section{FLAVOR IN THE LEPTONIC SECTOR OF THE LWSM}

The LWSM is usually formulated in terms of flavor eigenstates. The particle content of the fermion fields of the model is shown in Table 1. A generation index is omitted, however when along the text it is required to specify the generation, a corresponding sub-index will be added.

The Yukawa interactions mix the SM fields with the LW fields and are not diagonal in the generation space. In order to understand flavor issues in the LWSM, and to be able to determine which are the 'longest-lived' acausal states, it is suitable to work in the mass eigenstates basis. With this objective, in this Section we first show how a change of basis may isolate the Yukawa terms within each generation, and then how a second change of basis performed within each generation gives the final physical states. We finish with an analysis of the interactions resulting from these physical states .

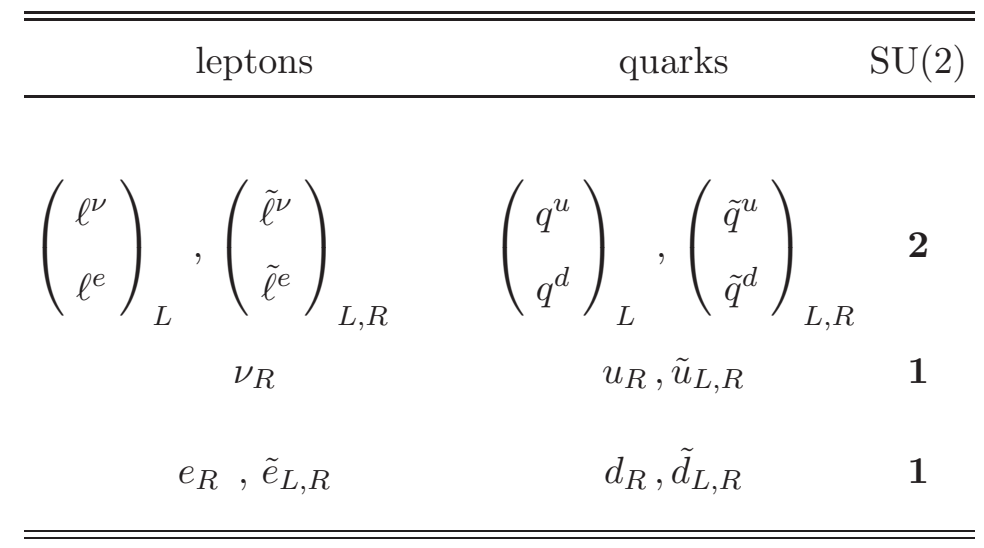

TABLE I: Fermionic fields in the LWSM. The neutrinos are Majorana particles. The right handed neutrino $\nu_{R}$ does not have a LW partner. The dimension of the $\mathrm{SU}(2)$ weak isospin representation is indicated in the last column. A generation subindex is omitted here, but it will be shown whenever necessary.

\section{A. Mixing between generations}

After the Higgs breaks the EW symmetry, the mass terms for the charged leptons in the LWSM are given by:

$$
\mathcal{L}_{e}=-\left(\bar{e}_{R}-\overline{\tilde{e}}_{R}\right) m_{e}^{\prime}\left(\ell_{L}^{e}-\tilde{\ell}_{L}^{e}\right)+\overline{\tilde{\ell}}_{R}^{e} M_{\ell} \tilde{\ell}_{L}^{e}+\overline{\tilde{e}}_{R} M_{e} \tilde{e}_{L}+\text { h.c. },
$$


where $M_{\ell, e}$ are the mass matrices of the LW lepton doublet and singlet, and $m_{e}^{\prime}=y_{e} v / \sqrt{2}$ is a $3 \times 3$ matrix in generation space, where generation indices are understood. Notice, for future purposes, that although the LW leptons are vector-like, only the Left- (Right-) chirality of the LW-doublet (singlet) is present in the the Yukawa interactions.

The Yukawa mixings between different generations can be diagonalized performing the following SM rotations:

$$
\begin{aligned}
e_{R} \rightarrow A_{R}^{e} e_{R}, & \tilde{e} \rightarrow A_{R}^{e} \tilde{e}, \\
\ell_{L}^{e} \rightarrow A_{L}^{e} \ell_{L}^{e}, & \tilde{\ell}^{e} \rightarrow A_{L}^{e} \tilde{\ell}^{e} ;
\end{aligned}
$$

where $A_{L, R}^{e}$ are the usual unitary matrices of the SM diagonalizing the Yukawa couplings of the charged leptons. Note that we have transformed both the Left- and Right-handed components of $\tilde{e}\left(\tilde{\ell}^{e}\right)$ with the same matrix $A_{R}^{e}\left(A_{L}^{e}\right)$. In this new basis the mass terms become:

$$
\mathcal{L}_{e}=-\left(\bar{e}_{R}-\overline{\tilde{e}}_{R}\right) m_{e}\left(\ell_{L}^{e}-\tilde{\ell}_{L}^{e}\right)+\overline{\tilde{\ell}}_{R}^{e} A_{L}^{e \dagger} M_{\ell} A_{L}^{e} \tilde{\ell}_{L}^{e}+\overline{\tilde{e}}_{R} A_{R}^{e \dagger} M_{e} A_{R}^{e} \tilde{e}_{L}+\text { h.c. }
$$

with $m_{e}$ the diagonal mass matrix of the SM. If we impose MFV in the LW sector, the matrices $M_{\ell, e}$ are proportional to the identity and we can trade $M_{\ell, e} \rightarrow M_{\ell, e} \mathbf{1}_{3 \times 3}$, with $M_{\ell, e}$ ordinary numbers. Therefore, Eq. (44) is diagonal in the generation space and one can consider the mixings between SM and LW leptons for each generation isolated from the others, as far as the mass terms are concerned ${ }^{5}$.

We consider now the neutral leptons of the LWSM. Ref. [10] showed that it is possible to preserve the familiar see-saw mechanism, without destabilizing the Higgs mass, by introducing a very heavy Right-handed neutrino, and no LW partner is needed for $\nu_{R}$. The corresponding mass Lagrangian is:

$$
\mathcal{L}_{\nu}=-\bar{\nu}_{R} m_{\nu}^{\prime}\left(\ell_{L}^{\nu}-\tilde{\ell}_{L}^{\nu}\right)-\frac{1}{2} \bar{\nu}_{R}^{c} m_{R} \nu_{R}+\overline{\tilde{\ell}}_{R}^{\nu} M_{\ell} \tilde{\ell}_{L}^{\nu}+\text { h.c. },
$$

with $m_{R}$ the Majorana mass of the Right-handed neutrino and a non-diagonal Dirac mass $m_{\nu}^{\prime}=y_{\nu} v / \sqrt{2}$. As before, the mixings between generations can be diagonalized with the

\footnotetext{
${ }^{5}$ If we relax MFV and allow for general $M_{\ell, e}$, it is not possible to diagonalize the mass matrix in the generation space with the usual SM transformation, and the mixings depend on the unknown parameters of the LW sector, see Ref. [8].
} 
usual SM transformations:

$$
\nu_{R} \rightarrow A_{R}^{\nu} \nu_{R}, \quad \ell_{L}^{\nu} \rightarrow A_{L}^{\nu} \ell_{L}^{\nu}, \quad \tilde{\ell}^{\nu} \rightarrow A_{L}^{\nu} \tilde{\ell}^{\nu}
$$

Note again that both chiralities of $\tilde{\ell}^{\nu}$ transform in the same way. Assuming MFV in the LW sector, the LW mass term remains diagonal. The only possible source of flavor mixing in the mass terms is the Majorana mass, and thus the generation mixings are suppressed by this very high scale.

We consider now the effect of the previous transformations in the interactions. Since the neutral current interactions are invariant under those transformations, the interactions with the photon, the $Z$, the neutral Higgs and their LW partners do not change flavor. Particularly important for the phenomenology are the following terms:

$$
\begin{aligned}
\mathcal{L}_{N C}= & -\left(Z_{\mu}+\tilde{Z}_{\mu}\right)\left[g_{z}^{e_{L}}\left(\bar{\ell}_{L}^{e} \gamma^{\mu} \ell_{L}^{e}-\overline{\tilde{\ell}} \gamma^{\mu} \tilde{\ell}^{e}\right)+g_{z}^{e_{R}}\left(\bar{e}_{R} \gamma^{\mu} e_{R}-\overline{\tilde{e}} \gamma^{\mu} \tilde{e}\right)+g_{z}^{\nu}\left(\bar{\ell}_{L}^{\nu} \gamma^{\mu} \ell_{L}^{\nu}-\overline{\tilde{\ell}}^{\nu} \gamma^{\mu} \tilde{\ell}^{\nu}\right)\right] \\
& \mathcal{L}_{N Y}=-\frac{y_{e}}{\sqrt{2}}\left(\bar{e}_{R}-\overline{\tilde{e}}_{R}\right)(h-\tilde{h}+i \tilde{P})\left(\ell_{L}^{e}-\tilde{\ell}_{L}^{e}\right)-\frac{y_{\nu}}{\sqrt{2}} \bar{\nu}_{R}(h-\tilde{h}-i \tilde{P})\left(\ell_{L}^{\nu}-\tilde{\ell}_{L}^{\nu}\right)+\text { h.c. }
\end{aligned}
$$

where $g_{z}^{e_{L, R}}$ and $g_{z}^{\nu_{L}}$ are the $Z$ couplings of the SM leptons, $y_{e, \nu}$ are the diagonal Yukawa couplings, and $\tilde{h}(\tilde{P})$ stands for the neutral scalar (pseudoscalar) component of the LW-Higgs field.

As usual, the charged current (CC) interactions are flavor changing. In terms of the new fields we obtain:

$$
\begin{array}{r}
\mathcal{L}_{C C}=-\frac{g_{2}}{\sqrt{2}}\left(W_{\mu}^{+}+\tilde{W}_{\mu}^{+}\right)\left(\bar{\ell}_{L}^{\nu} \gamma^{\mu} V \ell_{L}-\overline{\tilde{\ell}}^{\nu} \gamma^{\mu} V \tilde{\ell}^{e}\right)+\text { h.c. }, \\
\mathcal{L}_{C Y}=y_{e}\left(\bar{e}_{R}-\overline{\tilde{e}}_{R}\right) V^{\dagger} \tilde{h}^{-}\left(\ell_{L}^{\nu}-\tilde{\ell}_{L}^{\nu}\right)-y_{\nu} \bar{\nu}_{R} V \tilde{h}^{+}\left(\ell_{L}^{e}-\tilde{\ell}_{L}^{e}\right)+\text { h.c. },
\end{array}
$$

where $V=A_{L}^{\nu \dagger} A_{L}^{e}$ is the usual Pontecorvo-Maki-Nakagawa-Sakata leptonic mixing matrix [27]. We can see from Eqs. (9) and (10) that, assuming MFV, all the flavor changing interactions are determined by the SM parameters.

\section{B. Mixings between SM and LW leptons}

The charged lepton mass eigenstates can be obtained by making a simplectic rotation for each generation, similar to the one performed in Ref. [17, 18]. For each generation, we define three dimensional flavor eigenvectors containing the SM charged lepton and their LW partners, $\tilde{e}$ and $\tilde{\ell}$ :

$$
E_{L}^{t}=\left(\ell_{L}^{e}, \tilde{e}_{L}, \tilde{\ell}_{L}^{e}\right), \quad E_{R}^{t}=\left(e_{R}, \tilde{e}_{R}, \tilde{\ell}_{R}^{e}\right)
$$


(note that we have changed the basis order compared with Refs. [17, 18]) and three dimensional mass eigenstate vectors:

$$
\mathcal{E}_{L}^{t}=\left(\mathcal{E}_{L}^{1}, \mathcal{E}_{L}^{2}, \mathcal{E}_{L}^{3}\right), \quad \mathcal{E}_{R}^{t}=\left(\mathcal{E}_{R}^{1}, \mathcal{E}_{R}^{2}, \mathcal{E}_{R}^{3}\right)
$$

related by a simplectic rotation in the following way:

$$
E_{L, R}=S_{L, R}^{e} \mathcal{E}_{L, R}
$$

Expanding in powers of Yukawa insertions, at leading order the mass eigenstates coincide with the flavor eigenstates, and the mixings are suppressed by powers of $m_{e} / M_{\ell, e}$,

$$
\mathcal{E}_{L, R}=E_{L, R}+\mathcal{O}\left(m_{e} / M_{\ell, e}\right)
$$

where $\mathcal{E}_{e, \mu, \tau}^{1}$ are the usual electron, muon and tau. We show the diagonalization using this approximation in the Appendix A.

The neutral lepton mass eigenstates are Majorana fermions, and all the mixings are suppressed by at least one power of $m_{R}$. Similarly to the charged leptons, we define a vector containing four Majorana neutrinos for each generation:

$$
N^{t}=\left(\ell_{L}^{\nu}+\ell_{L}^{\nu c}, \nu_{R}+\nu_{R}^{c}, \tilde{\ell}_{L}^{\nu}+\tilde{\ell}_{L}^{\nu c}, \tilde{\ell}_{R}^{\nu}+\tilde{\ell}_{R}^{\nu c}\right)
$$

and a four dimensional mass eigenstate vector:

$$
\mathcal{N}^{t}=\left(\mathcal{N}^{1}, \mathcal{N}^{2}, \mathcal{N}^{3}, \mathcal{N}^{4}\right)
$$

related by a simplectic rotation:

$$
N=S^{\nu} \mathcal{N}
$$

There is a light neutrino $\mathcal{N}^{1}$, whose mass is given, at leading order in Yukawa insertions, by the usual see-saw mechanism. There are two neutrinos $\mathcal{N}^{3,4}$ that can be associated with the degrees of freedom of $\tilde{\ell}^{\nu}$, with masses $M_{\ell}+\mathcal{O}\left(m_{\nu}\right)$, and a fourth heavy neutrino $\mathcal{N}^{2}$ that can be associated with $\nu_{R}$, up to corrections of order $\sim \mathcal{O}\left(\frac{m_{\nu}}{m_{R}}\right)$. We show the details of the diagonalization in the Appendix A. As an interesting aspect of this result, we notice that the usual see-saw mechanism is not destabilized by the addition of a LW neutrino with $\sim$ TeV mass. 


\section{Interaction features of the mass eigenstates}

To obtain the interactions between the mass eigenstates one has to perform the above simplectic rotations of the charged and neutral leptons in the interaction terms. This can be done immediately by using the rotation matrices of Appendix A. The details of this calculation are found in Appendix B, where we explicitly show the interactions between the mass eigenstates.

Once we have the interactions of the mass eigenstates its decay properties are analyzed straightforward. For the purposes we follow, we are interested in the decay of the acausal charged and neutral leptons $\mathcal{E}^{2,3}$ and $\mathcal{N}^{3,4}$.

We begin analyzing neutral currents (NC). These interactions do not change flavor, however they can produce the interaction between different mass eigenstates. This is not the case for the electromagnetic interaction, since the SM leptons and their LW partners have the same electromagnetic charge and hence heavy leptons can not decay at tree level electromagnetically. On the other hand, the interactions with $Z$ (and $\tilde{W}^{3}$ and $\tilde{B}$ ) mix different mass eigenstates within a given generation because not all the leptons with a given chirality have the same $Z$-coupling, for example: $\tilde{e}_{L}$, and $\ell_{L}^{e}$ and $\tilde{\ell}_{L}^{e}$, although being all Left-handed charged fermions -see Eq. (11) - have different Z-couplings. Therefore, the heavy charged leptons can decay via $\mathcal{E}_{2,3} \rightarrow Z+\mathcal{E}^{1}$, with a suppression factor $m_{e} / M_{\ell, e}$ in the amplitude. In the flavor basis this is understood as a Yukawa insertion times a suppressing LW-fermionic propagator, as shown in Fig. 3. The heavy neutral leptons can decay by a similar process to $Z+\mathcal{N}^{1}$, but in this case with an extra Majorana suppression $m_{\nu} / m_{R}$.

The neutral Higgs interactions (NY) mix mass eigenstates. The charged leptons $\mathcal{E}^{2,3}$ interact with the light leptons $\mathcal{E}^{1}$ with Yukawa strength $y_{e}$ and no extra suppression. The coupling between neutral leptons $\mathcal{N}^{3,4}$ and $\mathcal{N}^{1}$ is proportional to $y_{\nu}$, but it is suppressed by $m_{\nu} / m_{R}$ since a virtual $\nu_{R}$ is needed to generate this interaction.

The charged current interactions also mix mass eigenstates and in addition, as usual, different generations. The heavy charged leptons $\mathcal{E}^{2,3}$ can decay to $W+\mathcal{N}^{1}$, with a coupling constant proportional to the leptonic mixing matrix $V$. The dominant charged current decays have amplitudes suppressed by $m_{e} / M_{e}$ for $\mathcal{E}^{2}$ and by $m_{e}^{2} / M_{\ell, e}^{2}$ for $\mathcal{E}^{3}$, where the Yukawa insertion $m_{e}$ has the same generation index as the decaying lepton. It is interesting to notice that this different suppression factor could also be understood through the flavor 
basis point of view, where the key difference comes from the Yukawa couplings, since the Yukawa terms only couple $\tilde{\ell}_{L}^{e}$ to $\tilde{e}_{R}$. On the other hand, the amplitudes for the neutral leptons decay, $\mathcal{N}^{3,4} \rightarrow \mathcal{E}^{1}+W$, is proportional to the corresponding $V$ and is suppressed by $m_{e} / M_{e}$, but the Yukawa insertion $m_{e}$ has the generation index corresponding to the final lepton $\mathcal{E}^{1}$. This is a crucial difference, since the contribution from a tau as a final lepton enhances the LW-neutrinos width.

The interactions with the charged LW Higgs $\tilde{h}^{ \pm}(\mathrm{CY})$ mix mass eigenstates and different generations. The coupling which drives $\mathcal{E}^{2,3} \rightarrow \tilde{h}^{ \pm} \mathcal{N}^{1}$ is proportional to $y_{e}$ and $V$, but the one corresponding to $\mathcal{E}^{3}$ has an extra suppression $\sim m_{e} / M_{\ell, e}$. Again, this difference is traceable to the different chiralities of the LW fields entering into the Yukawa interactions. At leading order, the coupling in charge of $\mathcal{N}^{3,4} \rightarrow \tilde{h}^{ \pm} \mathcal{E}^{1}$ is proportional to the Yukawa of the final charged lepton, $y_{e}$, without extra suppressions. Again, the tau contribution will enhance the LW-neutrinos width through this channel.

Using Appendix B, we summarize the relevant interactions for the decay of the LW-mass eigenstates $\mathcal{E}^{2,3}$ and $\mathcal{N}^{3,4}$ in Table II.

\begin{tabular}{|c|c|c|c|c|}
\hline lepton|interaction & $\mathrm{NC}$ & $\mathrm{NY}$ & $\mathrm{CC}$ & $\mathrm{CY}$ \\
\hline $\mathcal{E}_{\beta}^{2}$ & $\frac{m_{e}^{\beta}}{M_{e}} \delta^{\alpha \beta}$ & $y_{e}^{\beta} \delta^{\alpha \beta}$ & $\frac{m_{e}^{\beta}}{M_{e}} V^{a \beta}$ & $y_{e}^{\beta} V^{a \beta}$ \\
\hline $\mathcal{E}_{\beta}^{3}$ & $\frac{m_{e}^{\beta}}{M_{\ell}} \delta^{\alpha \beta}$ & $y_{e}^{\beta} \delta^{\alpha \beta}$ & $\left(\frac{m_{e}^{\beta}}{M_{e, \ell}}\right)^{2} V^{a \beta}$ & $y_{e}^{\beta} \frac{m_{e}^{\beta}}{M_{e, \ell}} V^{a \beta}$ \\
\hline $\mathcal{N}_{b}^{3,4}$ & $\frac{\left(m_{\nu}^{b}\right)^{2}}{M_{\ell} m_{R}} \delta^{a b}$ & $y_{\nu}^{a}\left(\frac{m_{\nu}^{a}}{m_{R}} \mp \frac{m_{\nu}^{a}}{M_{\ell} \mp m_{R}}\right) \delta^{a b}$ & $\frac{m_{e}^{\alpha}}{M_{\ell}} V^{\dagger \alpha b}$ & $y_{e}^{\alpha} V^{\dagger \alpha b}$ \\
\hline
\end{tabular}

TABLE II: Relevant interactions for the decay of the LW leptons. We show explicitly the indices $a, b=1,2,3$ that number the generations of neutrino mass eigenstates, and $\alpha, \beta=e, \mu, \tau$ that correspond to flavor. $m_{e}^{\alpha}$ and $m_{\nu}^{a}$ stand for the corresponding Dirac masses: $m_{e}^{\alpha}=y_{e}^{\alpha} v / \sqrt{2}$ and $m_{\nu}^{a}=y_{\nu}^{a} v / \sqrt{2}$. For the NC interactions involving $\mathcal{E}^{i}$ or $\mathcal{N}^{i}$ and $Z$ we show only the decaying lepton in the first column, thus the couplings correspond to $\mathcal{E}_{\beta}^{2} \rightarrow Z+\mathcal{E}_{\alpha}^{1}, \mathcal{E}_{\beta}^{3} \rightarrow Z+\mathcal{E}_{\alpha}^{1}$ and $\mathcal{N}_{b}^{3,4} \rightarrow Z+\mathcal{N}_{a}^{1}$, for the first, second and third line respectively. A similar situation holds for the other interactions that drive the following decays: $\mathrm{NY}\left(\mathcal{E}_{\beta}^{2,3} \rightarrow h+\mathcal{E}_{\alpha}^{1}, \mathcal{N}_{b}^{3,4} \rightarrow h+\mathcal{N}_{a}^{1}\right)$, $\mathrm{CC}\left(\mathcal{E}_{\beta}^{2,3} \rightarrow W+\mathcal{N}_{a}^{1}, \mathcal{N}_{b}^{3,4} \rightarrow W+\mathcal{E}_{\alpha}^{1}\right), \mathrm{CY}\left(\mathcal{E}_{\beta}^{2,3} \rightarrow \tilde{h}+\mathcal{N}_{a}^{1}, \mathcal{N}_{b}^{3,4} \rightarrow \tilde{h}+\mathcal{E}_{\alpha}^{1}\right)$. This table is obtained from Appendix B results. 


\section{WIDTH OF NARROW LEE-WICK RESONANCES}

In this Section we explicitly compute the width of narrow LW resonances. As we will see, the magnitude of these widths will select the first generation $\mathcal{E}_{e}^{2}$ and $\mathcal{E}_{e}^{3}$-the subindex indicates the first generation- as the best candidates to produce wrong vertex displacements at the LHC. It is worth to mention that this study is based on the analysis of two-body decays, unless something different is stated.

In the previous Section we found that $\mathcal{E}_{\alpha}^{2,3}$ decays were suppressed by the Yukawa $y_{e}^{\alpha}$, that corresponds to the generation of the decaying LW particle. Therefore, as a general feature, the mass states associated to the first generation are more stable than the others. On the other hand, the dominant decays of $\mathcal{N}^{3,4}$ involve charged interactions that mix generations, and are proportional to the Yukawa of the final charged lepton. Hence, the tau channel dominates and gives a larger width for these LW neutrinos. We compute explicitly the decay width for the $\mathcal{E}^{2,3}$ and $\mathcal{N}^{3,4}$ LW-mass eigenstates of the first generation.

\section{A. $\mathcal{E}^{2}$ total width}

The NC decay $\mathcal{E}^{2} \rightarrow Z+\mathcal{E}^{1}$ is suppressed by $m_{e}^{2} / M_{e}^{2}$ (the partial width is proportional to the coupling square), without flavor change. A similar suppression factor is present for the decay through CC interactions, but in this case the final neutrino can have any flavor, with a coefficient given by $V$, that is near to tribimaximal mixing. The NY decay $\mathcal{E}^{2} \rightarrow h+\mathcal{E}^{1}$ is controlled by the Yukawa coupling, without flavor change and extra suppressions (and similar for $\tilde{h}$ and $\tilde{P}$, although we expect them to be heavier than the SM Higgs, and for this reason we will neglect them in the rest of our analysis). This channel is open if $M_{e}>m_{h}$, otherwise it proceeds through a virtual Higgs, giving a three body decay. The charged Yukawa coupling of the heavy fermion also drives the CY decay $\mathcal{E}^{2} \rightarrow \tilde{h}^{-}+\mathcal{N}^{1}$, with the flavor of the final neutrino controlled by $V$. As previously discussed, at leading order the ratios of the widths of $\mathcal{E}_{e}^{2}, \mathcal{E}_{\mu}^{2}$ and $\mathcal{E}_{\tau}^{2}$ are given by: $m_{e}^{2}: m_{\mu}^{2}: m_{\tau}^{2}$. Meaning that the electron-resonance, $\mathcal{E}_{e}^{2}$, is the most stable state in this group.

In Fig. 3 we show the Feynman diagrams corresponding to the different decay channels of $\mathcal{E}_{e}^{2}$ doing a perturbative expansion in the mass insertions.

In Fig. 4 a we show the partial widths of $\mathcal{E}_{e}^{2}$ as a function of $M_{\ell}$, for $M_{\ell} \simeq M_{e}$, with 

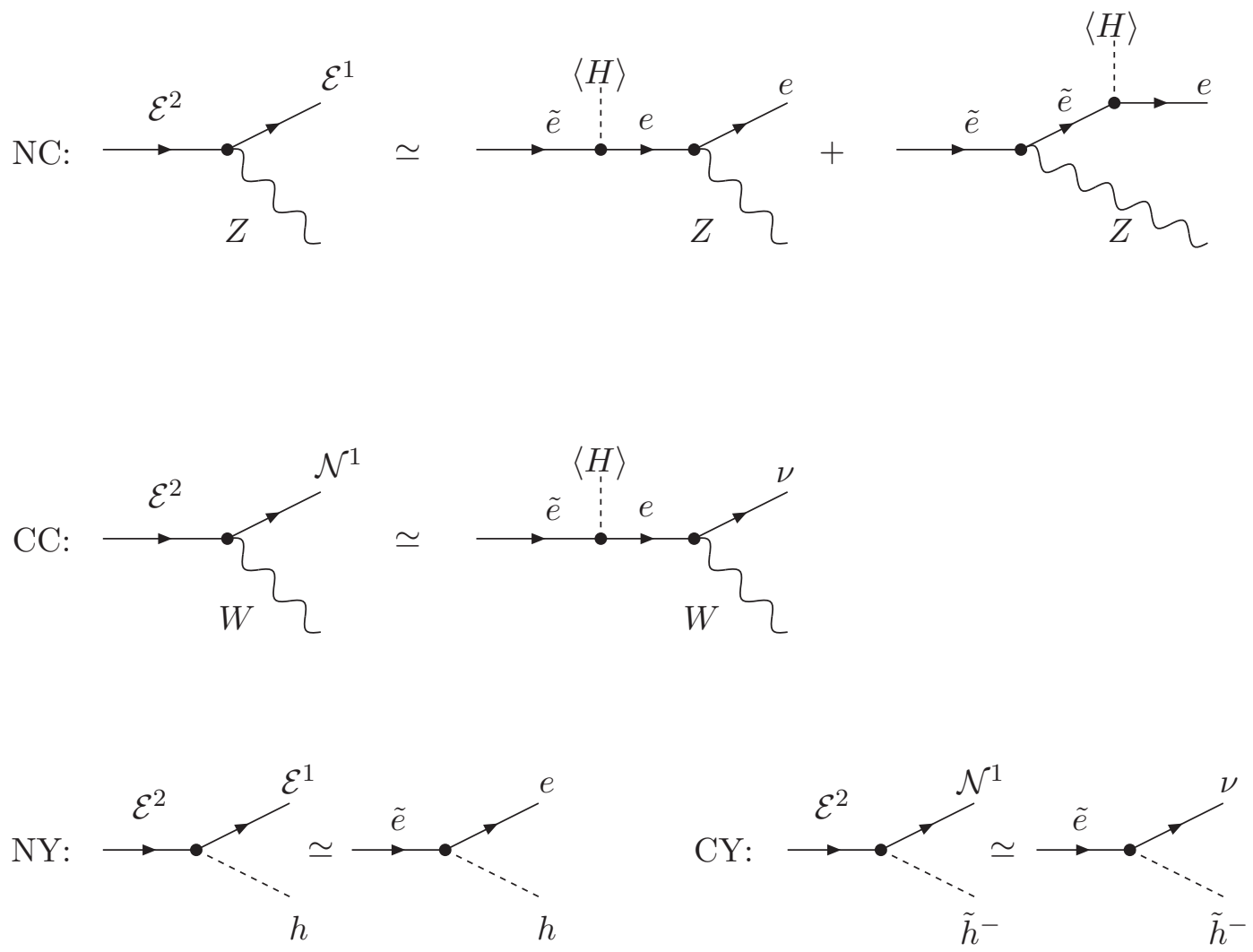

FIG. 3: Feynman diagrams for the decay channels of $\mathcal{E}^{2} \simeq \tilde{e}+\mathcal{O}\left(m_{e} / M_{\ell, e}\right)$, expanding in powers of mass insertions.

$m_{h}=200 \mathrm{GeV}$. We can see that the width is dominated by the charged current decay and for $M_{\ell}=300 \mathrm{GeV}(600,1000 \mathrm{GeV})$ the corresponding 'lifetime' is $\tau_{\mathcal{E}_{e}^{2}} \simeq 2 \cdot 10^{-14} \mathrm{~s}$ $\left(8 \cdot 10^{-15}, 4 \cdot 10^{-15} \mathrm{~s}\right)$.

\section{B. $\mathcal{E}^{3}$ total width}

From Table I we obtain that the decays of $\mathcal{E}^{3}$ are similar to those of $\mathcal{E}^{2}$, analyzed in the previous section, except that the charged channels have an extra suppression factor $m_{e} / M_{\ell, e}$ in the interactions. For this reason the decay of $\mathcal{E}_{e}^{3}$ is dominated by the neutral decay channels $\mathcal{E}_{e}^{3} \rightarrow Z+\mathcal{E}_{e}^{1}$ and $\mathcal{E}_{e}^{3} \rightarrow h+\mathcal{E}_{e}^{1}$, and its total with is smaller than the width of $\mathcal{E}_{e}^{2}$. For $m_{h}=200 \mathrm{GeV}$ and $M_{\ell} \lesssim 700 \mathrm{GeV}$ the $\mathrm{NC}$ channel dominates. In Fig. 4b we show the partial widths of $\mathcal{E}_{e}^{3}$ as a function of $M_{\ell}$, for $M_{\ell} \simeq M_{e}$, with $m_{h}=200 \mathrm{GeV}$. For $M_{\ell}=300$ $\mathrm{GeV}(600,1000 \mathrm{GeV})$ the corresponding 'lifetime' is $\tau_{\mathcal{E}_{e}^{3}}=4 \cdot 10^{-14} \mathrm{~s}\left(2 \cdot 10^{-14}, 9 \cdot 10^{-15} \mathrm{~s}\right)$. 


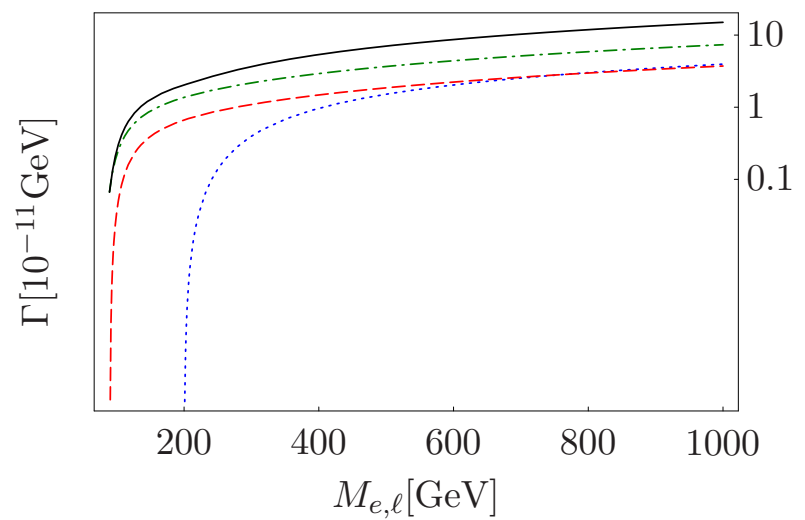

(a)

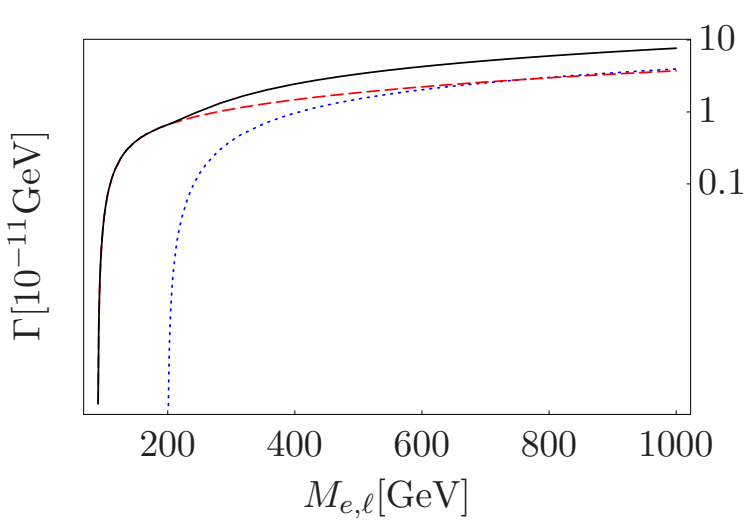

(b)

FIG. 4: (color online) (a) Partial widths of $\mathcal{E}_{e}^{2}$ as a function of $M_{e} \simeq M_{\ell}$ and $m_{h}=200 \mathrm{GeV}$. The dotted (blue) line corresponds to $\Gamma\left[\mathcal{E}_{e}^{2} \rightarrow h+\mathcal{E}_{e}^{1}\right]$, the dashed (red) line to $\Gamma\left[\mathcal{E}_{e}^{2} \rightarrow Z+\mathcal{E}_{e}^{1}\right]$, the dotted-dashed (green) line to $\Gamma\left[\mathcal{E}_{e}^{2} \rightarrow W+\mathcal{N}^{1}\right]$, and the solid (black) line to the total width. The CC decay dominates the width. (b) Partial widths of $\mathcal{E}_{e}^{3}$ as a function of $M_{e} \simeq M_{\ell}$ and $m_{h}=200 \mathrm{GeV}$. The dotted (blue) line corresponds to $\Gamma\left[\mathcal{E}_{e}^{3} \rightarrow h+\mathcal{E}_{e}^{1}\right]$, the dashed (red) line to $\Gamma\left[\mathcal{E}_{e}^{3} \rightarrow Z+\mathcal{E}_{e}^{1}\right]$ and the solid (black) line to the total width. The NC decay dominates the width for $M_{\ell} \lesssim 700 \mathrm{GeV}$ if $m_{h} \gtrsim 200 \mathrm{GeV}$.

There is an important three-body decay channel that could increase the width of $\mathcal{E}^{3}$ : $\mathcal{E}_{e}^{3} \rightarrow W^{-} W^{+} \mathcal{E}_{\tau}^{1}$. At a perturbative level, this process is given by the decay $\tilde{\ell}_{e}^{e} \rightarrow \tilde{\ell}_{a}^{\nu} W^{-} \rightarrow$ $W^{-} W^{+} \tau$, with a virtual $\tilde{\ell}_{a}^{\nu}$ and a suppression factor $m_{\tau} / M_{\ell}$ (much larger than the usual $\left.m_{e} / M_{\ell}\right)$. However, in the MFV scenario, this process is forbidden by a GIM-like mechanism.

\section{C. $\mathcal{N}^{3,4}$ total width}

The decay of $\mathcal{N}^{3,4}$ through neutral channels is suppressed by a Majorana mass $\sim m_{\nu}^{2} / m_{R}^{2}$, as shown in Table II. If the small neutrino masses are generated by the see-saw mechanism, these decay channels have a huge suppression and can be neglected. On the other hand, the decay through charged current interactions, $\mathcal{N}_{b}^{3,4} \rightarrow W+\mathcal{E}_{\alpha}^{1}$, with $\alpha=e, \mu, \tau$, is suppressed by $V^{\dagger \alpha b} m_{e}^{\alpha} / M_{\ell}$. Let us consider the decay of the heavy neutrinos $\mathcal{N}_{1}^{3,4} \rightarrow W+\mathcal{E}_{\tau}^{1}$, since neither of the matrix elements $V^{\dagger \alpha 1}$ is small, this channel is suppressed by $m_{\tau}^{2} / M_{\ell}^{2}$ only, giving rise to a rather large partial width $\sim 2 \cdot 10^{-4} \mathrm{GeV}$ for $M_{\ell} \sim 300 \mathrm{GeV}$. A similar 
thing happens with the other mass eigenstates $\mathcal{N}_{2,3}^{3,4}$. The decay through charged LW-Higgs interactions is also flavor changing, and for this reason the dominant channel is proportional to the $\tau$ Yukawa. Thus, due to the large mass of the $\tau$ and the large flavor mixings in the leptonic sector of the SM, the neutral heavy leptons have a small 'lifetime' $\sim 10^{-25} \mathrm{~s}$, and we do not expect them to produce vertex displacements.

\section{EXPERIMENTAL PERSPECTIVES}

From the previous Section we conclude that the best candidate to produce an observable wrong vertex displacement is the LW electron associated to the $\mathrm{SU}(2)$ doublet, $\mathcal{E}_{e}^{3} \simeq \tilde{\ell}_{e}^{e}+\mathcal{O}\left(m_{e} / M_{\ell, e}\right)$, since it is expected to have the smallest width, or largest 'lifetime'. For notational simplicity in the discussion that follows we will refer to the mass eigenstate

$\mathcal{E}_{e}^{3}$ and $\mathcal{E}_{e}^{1}$ as $\tilde{\ell}_{e}^{e}$ and $e$, respectively. We study now the production and detection of a pair of charged LW electrons in colliders like LHC and ILC. First, we enumerate a set of conditions we pursue in order to achieve a clear identification of events arising from the decay of these particles.

- The LW electrons will be mostly produced in pairs (single production is suppressed by a factor $\left.m_{e} / M_{\ell, e}\right)$ via EW interactions ${ }^{6}$ :

$$
q \bar{q} \rightarrow A, Z, \tilde{B}, \tilde{W}^{3} \rightarrow \overline{\tilde{\ell}}_{e}^{e} \tilde{\ell}_{e}^{e}
$$

Thus, we require a pair of correlated LW electrons, meaning that both of them are created in the same single primary vertex. Since all the LW interactions are determined by the SM couplings, the production cross section only depends on the value of the LW masses.

- Under the MFV hypothesis, the $\tilde{\ell}_{e}^{e}$ mainly decays -through neutral interactions- into an electron and a $Z$ or a Higgs boson, which in turn can decay into a fermion pair. For a reasonable Higgs mass $115 \mathrm{GeV}<m_{h}<300 \mathrm{GeV}$ and a $\mathrm{LW}$ mass $100 \mathrm{GeV}<$

\footnotetext{
${ }^{6}$ We have not taken into account mixings in the gauge-boson sector at this stage since they turn out to be highly suppressed $\left(\mathrm{M}_{(\mathrm{W}, \mathrm{Z})}^{2} / \mathrm{M}_{(\tilde{\mathrm{W}}, \tilde{\mathrm{B}})}^{2}\right)$ and we do not expect to have significant variations in the process we are considering.
} 
$M_{\ell}<1000 \mathrm{GeV}$, either the $Z$ channel dominates the decay or is halved by the Higgs channel. Assuming a $Z$ channel for the $\tilde{\ell}_{e}^{e}$ decay, Eq. (18) leads to:

$$
\overline{\tilde{\ell}}_{e} \tilde{\ell}_{e}^{e} \rightarrow Z e^{+} Z e^{-} .
$$

Each $Z$ can decay hadronically or leptonically, leading to a final state with an electronpositron pair and in addition: (i) four jets, (ii) two jets and a lepton-antilepton pair, or (iii) two lepton-antilepton pairs, depending on whether both, one or none of the $Z$ decays hadronically.

- We will require that the traces corresponding to one of the electrons and two of the jets (or one electron and one lepton-antilepton pair) converge in a vertex well separated from another vertex defined by the extrapolation of the traces of the remaining electron and a pair of jets. The relative position of these secondary vertices in the transverse plane is such that it brings forward the presence of two wrong vertex displacements and it is essential in the positive identification of acausal resonances. We will return to this point below.

- A fourth requirement is related to the measurement of the invariant mass corresponding to the decay products of the two LW electrons. We will see below that the reconstruction of the LW-electron mass is a necessary condition to distinguish acausal from causal resonances. This can be obtained by measuring the invariant mass of the three particles emerging from each displaced vertex, as explained in the previous paragraph.

The resulting physical situation corresponds to the one illustrated in Fig. 2. Projecting that picture onto the transverse plane, we can obtain the unusual position of the vertices in relation to the traces associated to them: traces of particles that go into the lower half plane converge from the vertex located in the upper region, and vice versa (this is what we stated as the essential condition in the third item). We see that the resulting momentum of the decay products is directed from the secondary towards the primary vertex.

Returning to the fourth item, we understand why it is necessary to reconstruct the LWelectron mass. For instance, if it turns out that some of the product particles is not detected (missing energy), it could happen that the true resulting momentum of the decay products points in the opposite direction (i.e., from the primary to the secondary vertex), as it would 
happen in the causal case ${ }^{7}$. By measuring the invariant mass of the decay products it is possible to distinguish among both situations.

\section{A. Displaced vertices at the LHC}

We briefly discuss the production and detection of LW-charged lepton pairs at the LHC. As already explained, since we look for displaced vertices away from the primary vertex, we will require the transverse displacement to be larger than a reference value that we take as $\Delta x=20 \mu \mathrm{m}$. For a particle with mass $M$ and transverse speed $v_{T}$, demanding it to travel a distance larger than $\Delta x$ results in the condition $v_{T} \gamma \tau>\Delta x$, where $\gamma$ and $\tau$ are its relativistic factor and lifetime. This is equivalent to the following condition on the transverse momentum: $p_{T}>M \Delta x \Gamma$, where $\Gamma$ is the total width of the resonance. This rough estimate allows us to obtain an approximate minimum transverse momentum $p_{T}$ for the LW resonance as a function of its mass. In the case of the LW-charged lepton associated to the $\mathrm{SU}(2)$ doublet, $\tilde{\ell}_{e}^{e}$, we obtain the following cuts in its transverse momentum:

$$
M_{\ell}=\left\{\begin{array} { l } 
{ 3 0 0 \mathrm { GeV } } \\
{ 4 0 0 \mathrm { GeV } } \\
{ 5 0 0 \mathrm { GeV } }
\end{array} \Longrightarrow \quad p _ { T } \gtrsim \left\{\begin{array}{c}
450 \mathrm{GeV} \\
980 \mathrm{GeV} \\
1700 \mathrm{GeV}
\end{array}\right.\right.
$$

Thus, only for light LW leptons we expect to obtain a sensible cross section after we demand a minimum distance between the primary and secondary vertices.

We used MadGraph/MadEvent [23] to obtain the total production cross section of a LW-charged lepton pair at the LHC with a center of mass energy of $14 \mathrm{TeV}$. We have also computed the cross-section after a cut in $p_{T}$ such that the transverse vertex displacement is greater than $\Delta x=20 \mu \mathrm{m}$. Fig. 5 shows a simulation for the $p_{T}$ distribution and its cut in $\overline{\tilde{\ell}}^{e} \tilde{\ell}^{e}$ production at the LHC. We plot our results for a relevant range of LW masses in Fig. 6, where we have taken $m_{h}=200 \mathrm{GeV}$. These results are slightly suppressed if $m_{h}$ is lower, see Table III. As a general feature we see that the cross section after the cut has a strong dependence with the LW scale. For $M_{\ell}=300 \mathrm{GeV}$ the total cross section is rather large,

\footnotetext{
7 This may occur in decays of supersymmetric particles. For example, if we consider the process $\tilde{t} \rightarrow t G$, the visible stop decay products may recoil against the invisible Goldstino in a direction towards the primary vertex [22].
} 
$\sim 66 \mathrm{fb}$, and after the cut we still have a sensible cross section $\sim 11 \mathrm{fb}$ for $m_{h}=200 \mathrm{GeV}$ and $\sim 8 \mathrm{fb}$ for $m_{h}=150 \mathrm{GeV}$. On the other hand, for $M_{\ell} \gtrsim 500 \mathrm{GeV}$, there is not enough energy at the LHC to create the highly boosted LW leptons.

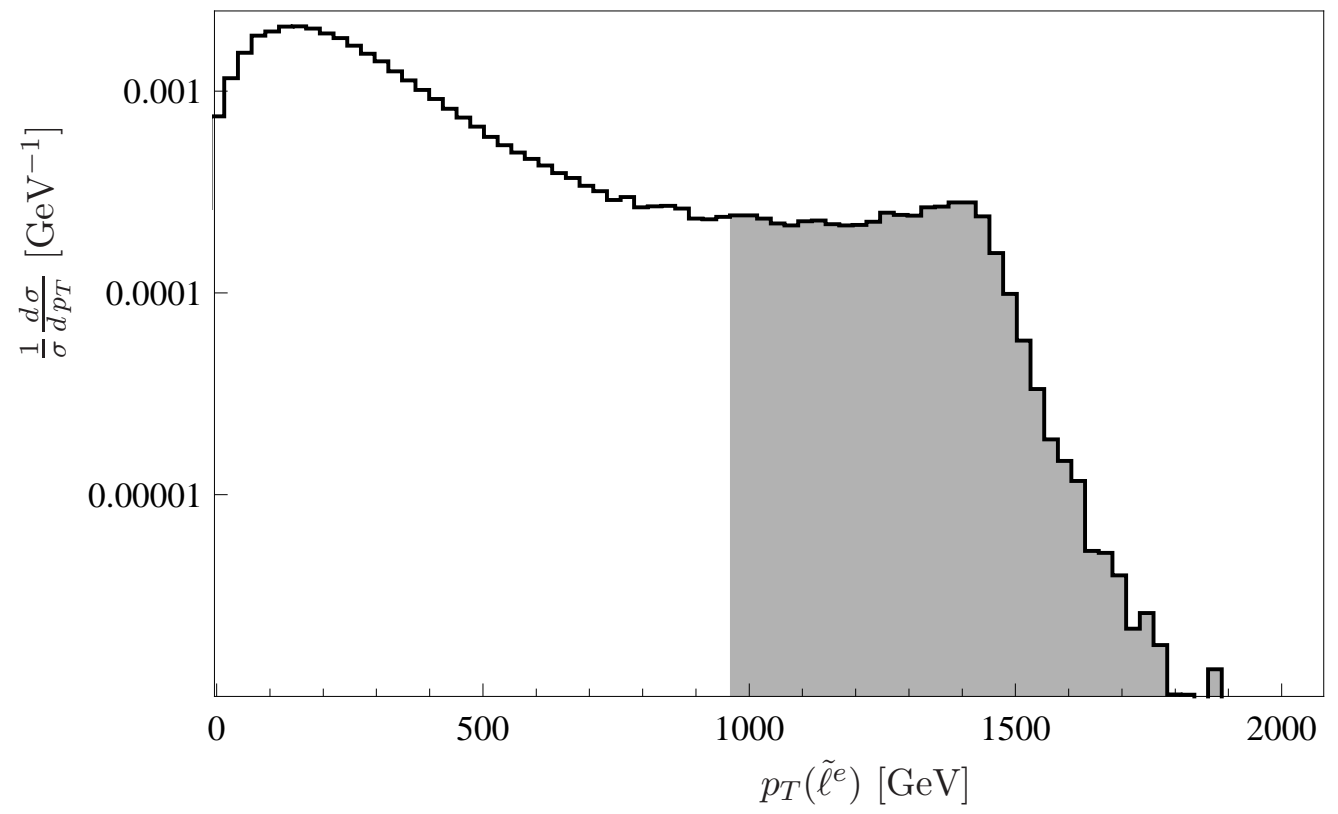

FIG. 5: $p_{T}$ distribution for $\overline{\tilde{\ell}}^{e} \tilde{\ell}^{e}$ production at the LHC setting $M_{\ell}=400 \mathrm{GeV}$. The shaded region -which survives after the cut in $p_{T^{-}}$represents the events that would produce wrong vertex displacements greater than $\Delta x=20 \mu \mathrm{m}$. We used $M_{\tilde{W}}=M_{\tilde{B}}=3 \mathrm{TeV}$ and a center of mass energy of $14 \mathrm{TeV}$. The first resonance corresponds to $Z$ and the second one to $\tilde{W}^{3}$ and $\tilde{B}$, whereas the tail for high $p_{T}$ is due to the lack of available energy in the quarks of the proton's beam.

Once created, each energetic LW lepton will mostly decay into a hard electron and $Z$ (we will consider $m_{h}=200 \mathrm{GeV}$ from now on). The electrons will have a very large $p_{T}$ because they are produced in a two body decay of a heavy LW lepton, and also because the LW state itself has a large $p_{T}$. For instance, for $M_{\ell}=200 \mathrm{GeV}$ the $p_{T}$ distribution of the final hardest electron is centered in $p_{T} \sim 200 \mathrm{GeV}$, whereas for $M_{\ell}=400 \mathrm{GeV}$ is centered in $p_{T} \sim 900 \mathrm{GeV}$, see Fig. 7 . Therefore, although the cut in the transverse momentum of the LW lepton suppresses the production cross section, at the same time, it allows us to impose hard cuts in the $p_{T}$ of the most energetic lepton, $p_{T} \gtrsim \mathcal{O}(200) \mathrm{GeV}$, with low impact in the signal. Each $Z$ gauge boson may decay leptonically or hadronically. The charged leptonic decay leads to a very clean final state, but has a small branching ratio, giving a cross section that is too small (unless the LW leptons are very light). Even the case where only one of 


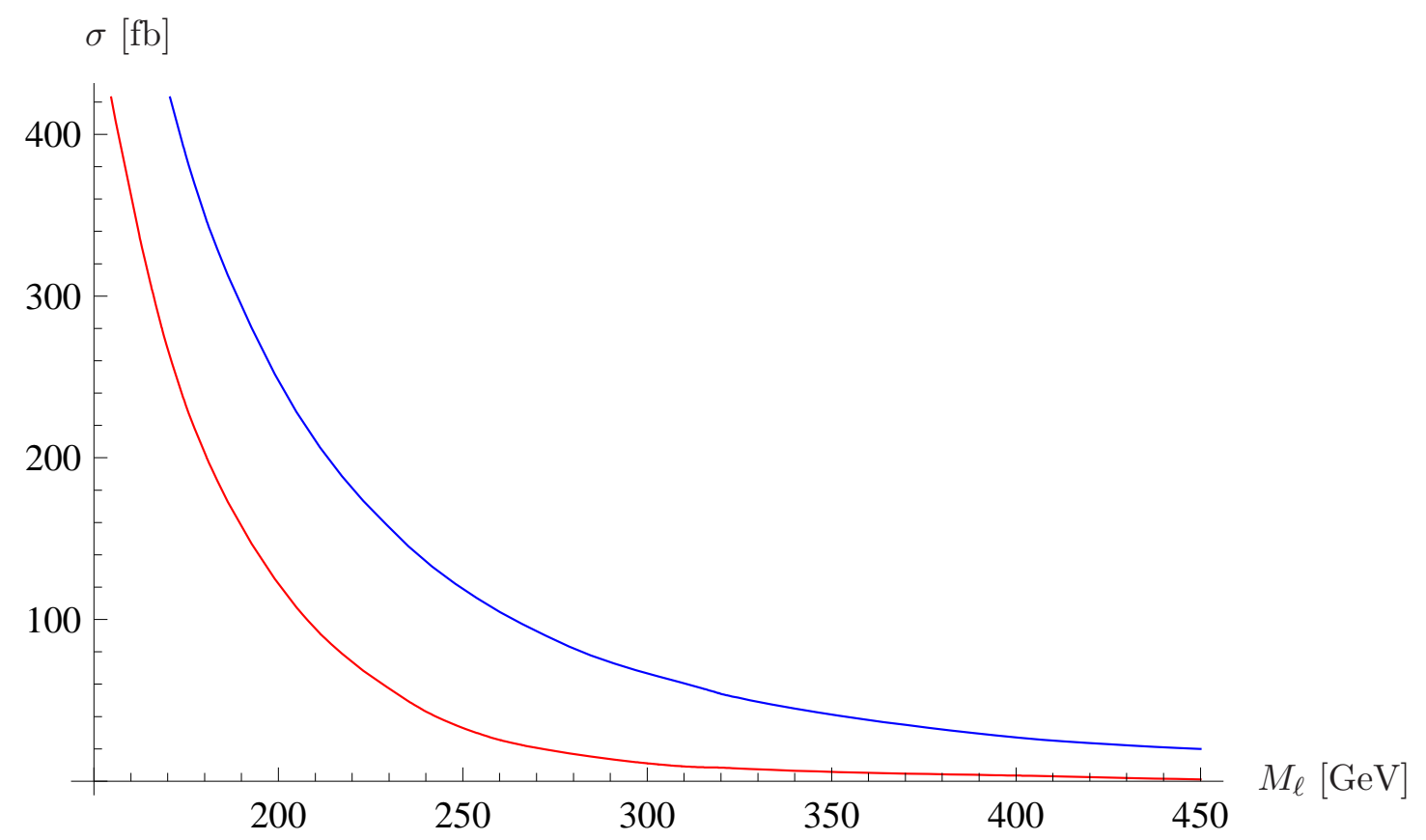

FIG. 6: Total (upper line) and $p_{T^{-c u t}}$ (lower line) cross-sections for $\overline{\tilde{\ell}}^{e} \tilde{\ell}^{e}$ production in LHC. The $p_{T}$ cut ensures that the wrong vertex displacement is greater than $\Delta x=20 \mu m$.

\begin{tabular}{|c|c|c|c|}
\hline$M_{\ell}[\mathrm{GeV}]$ & $\sigma_{t}[\mathrm{fb}]$ & $\left.\sigma_{\mathrm{cut}}\right|_{m_{h}=200 \mathrm{GeV}}[\mathrm{fb}]$ & $\left.\sigma_{\mathrm{cut}}\right|_{m_{h}=150 \mathrm{GeV}}[\mathrm{fb}]$ \\
\hline 300 & 66.7 & 11 & 8.4 \\
\hline 400 & 27 & 3.5 & 2.6 \\
\hline 500 & 15 & - & - \\
\hline
\end{tabular}

TABLE III: Cross section for the production of a LW-lepton pair at the LHC, simulated with MadGraph/MadEvent. The second column, $\sigma_{t}$, is the total cross section. The third and fourth columns correspond to the cross sections after the cut in the transverse momentum for $m_{h}=200$ $\mathrm{GeV}$ and $m_{h}=150 \mathrm{GeV}$, respectively, needed to obtain an observable displaced vertex. The empty box corresponds to a cross section $\sigma \lesssim \mathcal{O}\left(10^{-3}\right) \mathrm{fb}$.

the $Z$ bosons decays leptonically may be out of reach, due to the small production cross section after the cut (20), see Table III. The hadronic $Z$ decay dominates, producing a jet pair for each vector. (Choosing this decay channel reduces the cross section of Table III] by approximately 50\%.) Therefore, the signal corresponding to the dominating channel is 
defined by a very energetic electron-positron pair and four jets:

$$
e^{+} e^{-} j j j j .
$$

The intermediate $Z$ will also have a large $p_{T}$, and could give rise to collinear jets, with small angular separation. In this case both jets could be resolved as a single jet. Each pair of jets with an invariant mass corresponding to the $Z$, when considered together with the proper lepton $e^{ \pm}$, will have an invariant mass peaked around the LW-lepton mass.

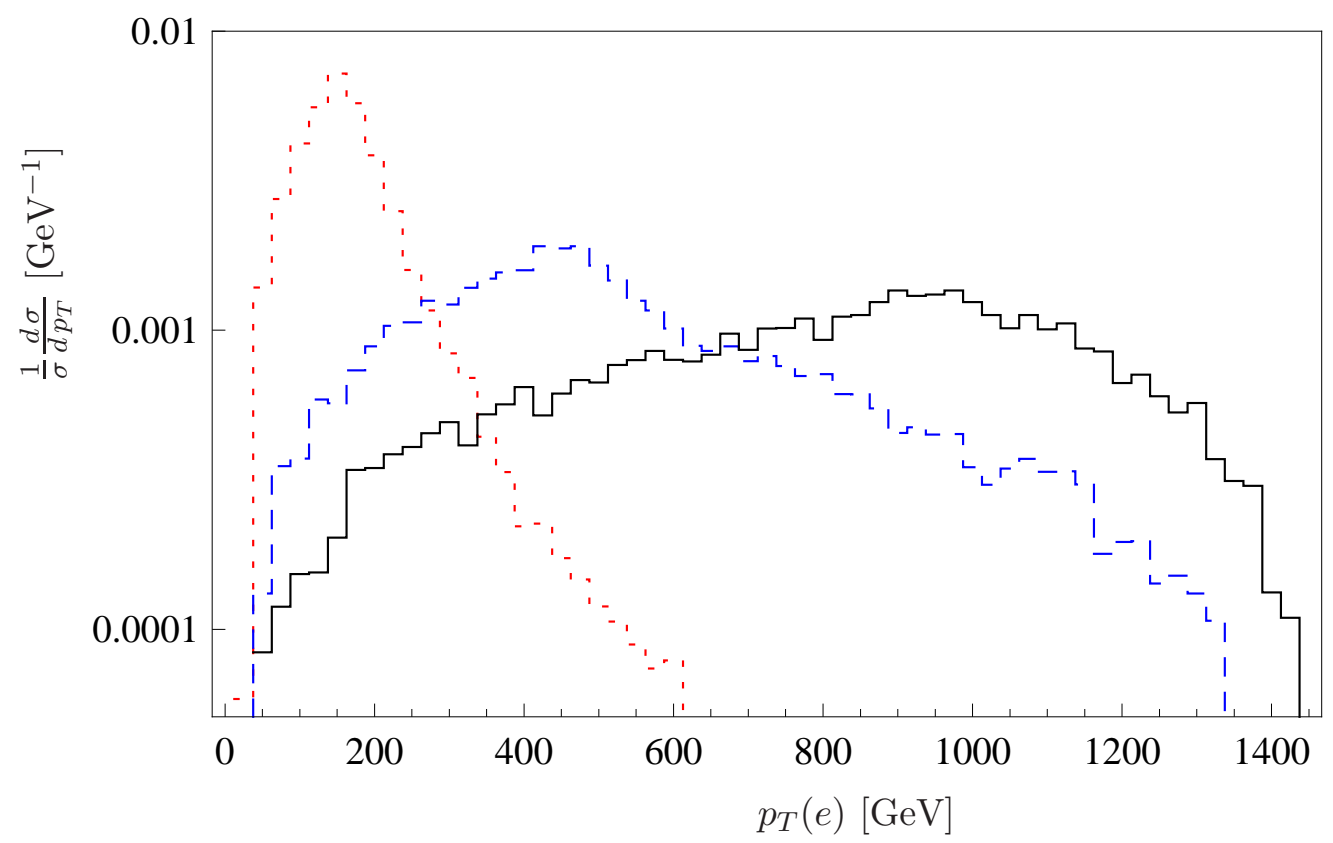

FIG. 7: (color online) $p_{T}$ distribution for the most energetic electron in the signal $e^{+} e^{-} j j j j$ after imposing the cut on the $p_{T}$ of the LW-leptons to obtain wrong vertex displacements greater than $\Delta x=20 \mu m$. The dotted (red), dashed (blue) and black (solid) lines correspond to LW masses $M_{\ell}=200 \mathrm{GeV}, 300 \mathrm{GeV}$ and $400 \mathrm{GeV}$, respectively.

The backgrounds for the signal here presented do not seem to cause further complications. However, a detailed SM background analysis should be performed to verify that the whole luminosity for the eejjjj signal data -with a suitable cut in the electrons' transverse momentum of $p_{T}(e)>200 \mathrm{GeV}$ - can be collected to perform an off-line analysis (this is known as an un-prescaled trigger). Afterwards, the peculiarity of this signal would need a proper vertexing algorithm to cope with the correct wrong vertex assignment. 


\section{CONCLUSIONS}

In this article we have defined a new signature called wrong vertex displacement which is a distinctive and model independent signal for new acausal resonances. Microscopic violation of causality is a feature of the Lee-Wick Standard Model [1], a well motivated model that can solve the hierarchy problem of the SM. We have proposed to use this new observable to detect acausal LW resonances at the LHC.

In order to determine whether the LW particles can produce measurable vertex displacements, we have made a detailed analysis of flavor in the leptonic sector of the LWSM. We obtained that assuming MFV the dominant decay channels of the charged LW leptons $\tilde{\ell}_{e}^{e}$ and $\tilde{e}_{e}$ are suppressed by the small charged Yukawa couplings, leading to tiny widths for the first generation of LW electrons (relaxing MFV in the leptonic sector leads to much larger widths, thus we do not expect to obtain measurable vertex displacements in that case). For LW lepton masses of the same order, $M_{\ell} \sim M_{e}$, the best LW candidate to produce a wrong vertex displacement at the LHC is the LW electron associated to the SU(2) doublet, $\tilde{\ell}_{e}^{e}$.

The LW electron singlet $\tilde{e}_{e}$ could also produce measurable wrong displaced vertices, although the production cross section is somewhat smaller due to the larger width compared with the previous case. The $\tilde{e}_{e}$ decays predominantly through $\mathrm{CC}$ interactions to $W \nu_{i}$, leading to a final state with large missing energy and making it impossible to reconstruct the mass of the intermediate resonances.

We have performed a Monte Carlo simulation and computed the cross-section to pair produce $\tilde{\ell}_{e}^{e}$ s which could generate wrong vertex displacement greater than $\Delta x=20 \mu m$. Our result is that for LW-leptonic masses satisfying $M_{\ell} \lesssim 450 \mathrm{GeV}$ it would be possible to observe wrong vertex displacements in the LHC era. The most promising final state is defined by $e^{+} e^{-} j j j j$, with highly boosted electrons and the jets coming from the hadronic decay of a pair of $Z$ gauge bosons. This final state allows the reconstruction of the LWleptonic masses. Although a full simulation analysis is needed, our study remarkably shows that low luminosities could confirm this signal: demanding five events would require an integrated luminosity of 1 (3) $\mathrm{fb}^{-1}$ for $M_{\ell}=300$ (400) GeV. The observation of this signal would point to the existence of acausal resonances, whereas its non-observation would not rule out models with this kind of particles, but could put lower bounds on the LW scale and give us information about the flavor structure of this particular model. 


\section{Acknowledgments}

We are grateful to Gustavo Otero y Garzón and Ricardo Piegaia for useful discussions on the experimental aspects of this article and Marcela Carena for valuable information. A.S. would like to thank Georges Azuelos and Hernán Wahlberg for helpful communications. The work of L.D. was supported by CONICET and partially supported by the State of São Paulo Research Foundation (FAPESP). The work of C.S. was supported by CONICET and partially supported by the U. S. Department of Energy, Office of Nuclear Physics under contract No. DE-FG02-93ER40756 with Ohio University.

\section{APPENDIX A: DIAGONALIZATION OF THE LEPTONIC MASS MATRICES}

In this section we diagonalize the leptonic mass matrices. We consider first the sector of charged leptons, using Eq. (4) and MFV we can disentangle the mixings between generations. Using Eq. (11) we can write the quadratic Lagrangian as:

$$
\mathcal{L}_{e}=i \bar{E} \not \partial \eta_{e} E-\bar{E}_{R} \mathcal{M}_{e} E_{L}-\bar{E}_{L} \mathcal{M}_{e}^{\dagger} E_{R}
$$

where $\eta_{e}=\operatorname{diag}(1,-1,-1)$ and

$$
\mathcal{M}_{e}=\left(\begin{array}{ccc}
m_{e} & 0 & -m_{e} \\
-m_{e} & -M_{e} & m_{e} \\
0 & 0 & -M_{\ell}
\end{array}\right) .
$$

The independent simplectic rotations $S_{L, R}$ defined by Eqs. (11,12,13) diagonalize $\mathcal{M}_{e}$ and satisfy the following relations:

$$
\mathcal{M}_{e, \mathrm{phys}}=S_{R}^{e \dagger} \mathcal{M}_{e} S_{L}^{e}, \quad S_{R}^{e} \eta_{e} S_{R}^{e \dagger}=\eta_{e}, \quad S_{L}^{e} \eta_{e} S_{L}^{e \dagger}=\eta_{e}
$$

where $\mathcal{M}_{e, \text { phys }}$ is the physical mass matrix, which is diagonal. Expanding in powers of $\epsilon_{e, \ell}=\frac{m_{e}}{M_{e, \ell}} \ll 1$ we obtain the following eigenvalues:

$$
\begin{aligned}
& m_{e}\left[1+\frac{1}{2}\left(\epsilon_{\ell}^{2}+\epsilon_{e}^{2}\right)+\frac{1}{8}\left(7 \epsilon_{\ell}^{4}+7 \epsilon_{e}^{4}+10 \epsilon_{\ell}^{2} \epsilon_{e}^{2}\right)\right]+\mathcal{O}\left(\epsilon_{\ell, e}^{6}\right), \\
& M_{e}\left[1-\frac{\epsilon_{e}^{2}}{2} \frac{M_{\ell}^{2}}{M_{\ell}^{2}-M_{e}^{2}}-\frac{\epsilon_{e}^{4}}{8} \frac{5 M_{\ell}^{6}-9 M_{\ell}^{4} M_{e}^{2}}{\left(M_{\ell}^{2}-M_{e}^{2}\right)^{3}}\right]+\mathcal{O}\left(\epsilon_{\ell, e}^{6}\right), \\
& M_{\ell}\left[1+\frac{\epsilon_{\ell}^{2}}{2} \frac{M_{e}^{2}}{M_{\ell}^{2}-M_{e}^{2}}+\frac{\epsilon_{\ell}^{4}}{8} \frac{5 M_{e}^{6}-9 M_{e}^{4} M_{\ell}^{2}}{\left(M_{\ell}^{2}-M_{e}^{2}\right)^{3}}\right]+\mathcal{O}\left(\epsilon_{\ell, e}^{6}\right) .
\end{aligned}
$$


and for $S_{L, R}^{e}$ we get:

$$
S_{L}^{e}-1=\left[\begin{array}{ccc}
\frac{\epsilon_{e}^{2}}{2} & -\epsilon_{e} & \frac{\epsilon_{\ell}^{4}}{\epsilon_{e}^{2}-\epsilon_{\ell}^{2}} \\
-\epsilon_{e} & \frac{\left.\epsilon_{e}^{4} \epsilon_{e}^{2}-2 \epsilon_{\ell}^{2}\right)}{2\left(\epsilon_{\ell}^{2}-\epsilon_{e}^{2}\right)^{2}} & \frac{\epsilon_{\ell}^{2} \epsilon_{e}}{\epsilon_{\ell}^{2}-\epsilon_{e}^{2}} \\
-\epsilon_{\ell}^{2} & \frac{\epsilon_{\ell}^{2} e_{e}}{\epsilon_{e}^{2}-\epsilon_{\ell}^{2}} & -\frac{\epsilon_{\ell}^{2} \epsilon_{e}}{2\left(\epsilon_{\ell}^{2}-\epsilon_{e}^{2}\right)^{2}}
\end{array}\right], \quad S_{R}^{e}-1=\left[\begin{array}{ccc}
\frac{\epsilon_{\ell}^{2}}{2} & \frac{\epsilon_{e}^{4}}{\epsilon_{\epsilon}^{2}-\epsilon_{e}^{2}} & -\epsilon_{\ell} \\
-\epsilon_{e}^{2} & \frac{\epsilon_{\ell}^{2} \epsilon_{e}^{4}}{2\left(\epsilon_{\ell}^{2}-\epsilon_{e}^{2}\right)^{2}} & \frac{\epsilon_{\epsilon} \epsilon_{e}^{2}}{\epsilon_{\ell}^{2}-\epsilon_{e}^{2}} \\
-\epsilon_{\ell} & \frac{\epsilon_{\ell} \epsilon_{e}^{2}}{\epsilon_{e}^{2}-\epsilon_{\ell}^{2}} & \frac{\epsilon_{\ell}^{(}\left(\epsilon_{\ell}^{2}-2 \epsilon_{e}^{2}\right)}{2\left(\epsilon_{\ell}^{2}-\epsilon_{e}^{2}\right)^{2}}
\end{array}\right] \text {. }
$$

The solution for the second and third generations is obtained exchanging the index $e$ by $\mu$ or $\tau$. This solution is valid for $M_{\ell} \neq M_{e}$, the solution for $M_{\ell}=M_{e}$ can be obtained in a similar way.

We consider now the sector of neutral leptons. Using Eqs. (5) and (6) and imposing MFV we can disentangle the generation mixing. From Eq. (15) we can write the quadratic Lagrangian as:

$$
\mathcal{L}_{\nu}=\frac{i}{2} \bar{N} \not \partial \eta_{\nu} N-\frac{1}{2} \bar{N} \mathcal{M}_{\nu} N,
$$

where $\eta_{\nu}=\operatorname{diag}(1,1,-1,-1)$ and

$$
\mathcal{M}_{\nu}=\left(\begin{array}{cccc}
0 & m_{\nu} & 0 & 0 \\
m_{\nu} & m_{R} & -m_{\nu} & 0 \\
0 & -m_{\nu} & 0 & -M_{\ell} \\
0 & 0 & -M_{\ell} & 0
\end{array}\right)
$$

The simplectic rotation matrix $S^{\nu}$, that diagonalizes $\mathcal{M}_{\nu}$, see Eq. (17), satisfies:

$$
\mathcal{M}_{\nu, \text { phys }}=S^{\nu \dagger} \mathcal{M}_{\nu} S^{\nu}, \quad S^{\nu} \eta_{\nu} S^{\nu \dagger}=\eta_{\nu},
$$

where $\mathcal{M}_{\nu \text {,phys }}$ is the physical mass matrix, which is diagonal. Expanding in powers of $m_{\nu}$, for $m_{\nu} \ll m_{R}, M_{\ell}$, we obtain the following eigenvalues:

$$
\begin{aligned}
m_{\nu, 1} & =\frac{m_{\nu}^{2}}{m_{R}}-\frac{m_{\nu}^{4}}{m_{R}^{3}}+\mathcal{O}\left(m_{\nu}^{6}\right) \\
m_{\nu, 2} & =m_{R}+m_{\nu}^{2} \frac{M_{\ell}^{2}}{m_{R}^{2}\left(M_{\ell}^{2}-m_{R}^{2}\right)}+m_{\nu}^{4} \frac{M_{\ell}^{4}\left(M_{\ell}^{2}-3 m_{R}^{2}\right)}{m_{R}^{3}\left(m_{R}^{2}-M_{\ell}^{2}\right)^{3}}+\mathcal{O}\left(m_{\nu}^{6}\right) \\
m_{\nu, 3} & =M_{\ell}+\frac{m_{\nu}^{2}}{2\left(m_{R}-M_{\ell}\right)}-m_{\nu}^{4} \frac{3 m_{R}-5 M_{\ell}}{M_{\ell}\left(m_{R}-M_{\ell}\right)^{3}}+\mathcal{O}\left(m_{\nu}^{6}\right) \\
m_{\nu, 4} & =M_{\ell}-\frac{m_{\nu}^{2}}{2\left(m_{R}+M_{\ell}\right)}-m_{\nu}^{4} \frac{3 m_{R}+5 M_{\ell}}{M_{\ell}\left(m_{R}+M_{\ell}\right)^{3}}+\mathcal{O}\left(m_{\nu}^{6}\right)
\end{aligned}
$$


and:

$$
S^{\nu}=\left[\begin{array}{cccc}
1-\frac{m_{\nu}^{2}}{2 m_{R}^{2}} & \frac{m_{\nu}}{m_{R}} & \frac{m_{\nu}^{2}}{\sqrt{2} M_{\ell}\left(M_{\ell}-m_{R}\right)} & \frac{m_{\nu}^{2}}{\sqrt{2} M_{\ell}\left(M_{\ell}+m_{R}\right)} \\
-\frac{m_{\nu}}{m_{R}} & 1-\frac{m_{\nu}^{2} M_{\ell}^{2}\left(M_{\ell}^{2}-3 m_{R}^{2}\right)}{2 m_{R}^{2}\left(m_{R}^{2}-M_{\ell}^{2}\right)^{2}} & \frac{m_{\nu}}{\sqrt{2}\left(M_{\ell}-m_{R}\right)} & -\frac{m_{\nu}}{\sqrt{2}\left(M_{\ell}+m_{R}\right)} \\
-\frac{m_{\nu}^{4}}{m_{R}^{2} M_{\ell}^{2}} & \frac{m_{\nu} m_{R}}{m_{R}^{2}-M_{\ell}^{2}} & -\frac{1}{\sqrt{2}}-\frac{m_{\nu}^{2} m_{R}}{4 \sqrt{2} M_{\ell}\left(M_{\ell}-m_{R}\right)^{2}} & -\frac{1}{\sqrt{2}}+\frac{m_{\nu}^{2} m_{R}}{4 \sqrt{2} M_{\ell}\left(M_{\ell}+m_{R}\right)^{2}} \\
\frac{m_{\nu}^{2}}{m_{R} M_{\ell}} & \frac{m_{\nu} M_{\ell}}{m_{R}^{2}-M_{\ell}^{2}} & -\frac{1}{\sqrt{2}}+\frac{m_{\nu}^{2}\left(m_{R}-2 M_{\ell}\right)}{4 \sqrt{2} M_{\ell}\left(M_{\ell}-m_{R}\right)^{2}} & \frac{1}{\sqrt{2}}+\frac{m_{\nu}^{2}\left(m_{R}+2 M_{\ell}\right)}{4 \sqrt{2} M_{\ell}\left(M_{\ell}+m_{R}\right)^{2}}
\end{array}\right]
$$

where we have written only the first non-trivial corrections for every entry of $S^{\nu}$. There is a similar solution for every generation, that can be obtained by considering the corresponding Dirac neutrino mass $m_{\nu}$.

\section{APPENDIX B: INTERACTIONS BETWEEN MASS EIGENSTATES}

In this section we show the leptonic interactions between the mass eigenstates. From Eqs. (7],13]17) we obtain the following neutral current interactions:

$$
\begin{aligned}
\mathcal{L}_{N C}=-\left(Z_{\mu}+\tilde{Z}_{\mu}\right) & {\left[g_{z}^{e_{L}} \overline{\mathcal{E}}_{L} \gamma^{\mu} \eta_{e} \mathcal{E}_{L}+g_{z}^{e_{R}} \overline{\mathcal{E}}_{R} \gamma^{\mu} \eta_{e} \mathcal{E}_{R}\right.} \\
+ & \left(g_{z}^{e_{L}}-g_{z}^{e_{R}}\right) \overline{\mathcal{E}}^{i} \gamma^{\mu}\left(S_{L, i 2}^{e \dagger} S_{L, 2 j}^{e} P_{L}-S_{R, i 3}^{e \dagger} S_{R, 3 j}^{e} P_{R}\right) \mathcal{E}^{j} \\
+ & \left.g_{z}^{\nu_{L}} \overline{\mathcal{N}}^{i} \gamma^{\mu}\left(S_{i 1}^{\nu \dagger} S_{1 j}^{\nu} P_{L}-S_{i 3}^{\nu \dagger} S_{3 j}^{\nu} P_{L}-S_{i 4}^{\nu \dagger} S_{4 j}^{\nu} P_{R}\right) \mathcal{N}^{j}\right],
\end{aligned}
$$

where $P_{L, R}$ are the Left and Right projectors, we have to sum over $i, j=1,2,3$ for the charged leptons and $i, j=1, \ldots 4$ for the neutral leptons, and a sum over a generation index is understood. We can see explicitly that the interactions between the charged leptons are not diagonal because $g_{z}^{e_{L}} \neq g_{z}^{e_{R}}$. The elements of the matrices $S^{e, \nu}$ can be read form Eqs. (A7) and (A15).

The neutral Higgs interactions are given by:

$$
\begin{aligned}
\mathcal{L}_{N Y}= & -\frac{y_{e}}{\sqrt{2}} \overline{\mathcal{E}}_{R}^{i}\left(S_{R, i 1}^{e \dagger}-S_{R, i 2}^{e \dagger}\right)(h-\tilde{h}+i \tilde{P})\left(S_{L, 1 j}^{e}-S_{L, 3 j}^{e}\right) \mathcal{E}_{L}^{j}+\text { h.c. } \\
& -\frac{y_{\nu}}{\sqrt{2}} \overline{\mathcal{N}}_{R}^{i} S_{i 2}^{\nu \dagger}(h-\tilde{h}-i \tilde{P})\left(S_{1 j}^{\nu}-S_{3 j}^{\nu}\right) \mathcal{N}_{L}^{j}+\text { h.c. }
\end{aligned}
$$

where a sum over a generation index is understood.

For the charged current and the charged LW-Higgs interactions we obtain:

$$
\begin{aligned}
\mathcal{L}_{C C}= & -\frac{g_{2}}{\sqrt{2}}\left(W_{\mu}^{+}+\tilde{W}_{\mu}^{+}\right) \overline{\mathcal{N}}^{i} \gamma^{\mu} V\left(S_{i 1}^{\nu \dagger} S_{L, 1 j}^{e} P_{L}-S_{i 3}^{\nu \dagger} S_{L, 3 j}^{e} P_{L}-S_{i 4}^{\nu \dagger} S_{R, 3 j}^{e} P_{R}\right) \mathcal{E}^{j}+\text { h.c. } \\
\mathcal{L}_{C Y}= & y_{\nu} \overline{\mathcal{N}}_{R}^{i} S_{i 2}^{\nu \dagger} V \tilde{h}^{+}\left(S_{L, 1 j}^{e}-S_{L, 3 j}^{e}\right) \mathcal{E}_{L}^{j}+\text { h.c. } \\
& +y_{e} \overline{\mathcal{E}}_{R}^{i}\left(S_{R, i 1}^{e \dagger}-S_{R, i 2}^{e \dagger}\right) V \tilde{h}^{-}\left(S_{1 j}^{\nu}-S_{3 j}^{\nu}\right) \mathcal{N}_{L}^{j}+\text { h.c. }
\end{aligned}
$$


where a sum over generations is understood.

[1] B. Grinstein, D. O'Connell and M. B. Wise, The Lee-Wick standard model, Phys. Rev. D 77 (2008) 025012 arXiv:0704.1845 [hep-ph]].

[2] T. D. Lee and G. C. Wick, Negative Metric and the Unitarity of the S Matrix, Nucl. Phys. B 9 (1969) 209.

[3] T. D. Lee and G. C. Wick, Finite Theory of Quantum Electrodynamics, Phys. Rev. D 2 (1970) 1033.

[4] B. Grinstein, D. O'Connell and M. B. Wise, Massive Vector Scattering in Lee-Wick Gauge Theory, Phys. Rev. D 77 (2008) 065010 [arXiv:0710.5528 [hep-ph]].

[5] Z. Fodor, K. Holland, J. Kuti, D. Nogradi and C. Schroeder, New Higgs physics from the lattice, PoS LAT2007 (2007) 056 [arXiv:0710.3151 [hep-lat]].

[6] F. Knechtli, N. Irges and M. Luz, New Higgs mechanism from the lattice, J. Phys. Conf. Ser. 110 (2008) 102006 arXiv:0711.2931 [hep-ph]].

[7] B. Grinstein and D. O'Connell, One-Loop Renormalization of Lee-Wick Gauge Theory, Phys. Rev. D 78 (2008) 105005 arXiv:0801.4034 [hep-ph]].

[8] T. R. Dulaney and M. B. Wise, Flavor Changing Neutral Currents in the Lee-Wick Standard Model, Phys. Lett. B 658 (2008) 230 [arXiv:0708.0567[hep-ph]].

[9] F. Wu and M. Zhong, The Lee-Wick Fields out of Gravity, Phys. Lett. B 659 (2008) 694 arXiv:0705.3287 [hep-ph]].

[10] J. R. Espinosa, B. Grinstein, D. O'Connell and M. B. Wise, Neutrino masses in the Lee-Wick standard model, Phys. Rev. D 77 (2008) 085002 [arXiv:0705.1188 [hep-ph]].

[11] T. G. Rizzo, Searching for Lee-Wick Gauge Bosons at the LHC, JHEP 0706 (2007) 070 arXiv:0704.3458 [hep-ph]].

[12] F. Krauss, T. E. J. Underwood and R. Zwicky, The process $g g \rightarrow h_{0} \rightarrow \gamma \gamma$ in the Lee-Wick Standard Model, Phys. Rev. D 77 (2008) 015012 arXiv:0709.4054 [hep-ph]].

[13] C. D. Carone and R. F. Lebed, Minimal Lee-Wick Extension of the Standard Model, Phys. Lett. B 668 (2008) 221 arXiv:0806.4555 [hep-ph]].

[14] T. G. Rizzo, Unique Identification of Lee-Wick Gauge Bosons at Linear Colliders, JHEP 0801 (2008) 042 [arXiv:0712.1791 [hep-ph]]. 
[15] C. D. Carone and R. F. Lebed, A Higher-Derivative Lee-Wick Standard Model, JHEP 0901 (2009) 043 arXiv:0811.4150 [hep-ph]].

[16] C. D. Carone, Higher-Derivative Lee-Wick Unification, Phys. Lett. B 677 (2009) 306 arXiv:0904.2359 [hep-ph]].

[17] E. Alvarez, L. Da Rold, C. Schat and A. Szynkman, Electroweak precision constraints on the Lee-Wick Standard Model, JHEP 0804 (2008) 026 arXiv:0802.1061 [hep-ph]]; E. Alvarez, C. Schat, L. Da Rold and A. Szynkman, Electroweak precision constraints on the Lee-Wick Standard Model, arXiv:0810.3463 [hep-ph].

[18] T. E. J. Underwood and R. Zwicky, Electroweak Precision Data and the Lee-Wick Standard Model, Phys. Rev. D 79 (2009) 035016 [arXiv:0805.3296 [hep-ph]].

[19] B. Grinstein, D. O'Connell and M. B. Wise, Causality as an emergent macroscopic phenomenon: The Lee-Wick O(N) model, Phys. Rev. D 79 (2009) 105019 arXiv:0805.2156 [hepth]].

[20] B. Fornal, B. Grinstein and M. B. Wise, Lee-Wick Theories at High Temperature, Phys. Lett. B 674 (2009) 330 arXiv:0902.1585 [hep-th]].

[21] S. Coleman, Acausality, In "Erice 1969, Ettore Majorana School On Subnuclear Phenomena", New York, 282 (1970).

[22] R. L. Culbertson et al. [SUSY Working Group Collaboration], Low scale and gauge mediated supersymmetry breaking at the Fermilab Tevatron Run II, arXiv:hep-ph/0008070.

[23] T. Sjostrand et al., High-energy physics event generation with PYTHIA 6.1, Comput. Phys. Commun. 135 (2001) 238 [hep-ph/0010017]; T. Sjostrand, High-energy physics event generation with PYTHIA 5.7 and JETSET 7.4, Comput. Phys. Commun. 82 (1994) 74.

[24] See, for instance, P. D. Acton et al. [OPAL Collaboration], Measurement of the average $b$ hadron lifetime in Z0 decays, Z. Phys. C 60 (1993) 217; J. Abdallah et al. [DELPHI Collaboration], A Precise measurement of the $B^{+}, B^{0}$ and mean $b$ hadron lifetime with the DELPHI detector at LEP1, Eur. Phys. J. C 33 (2004) 307 arXiv:hep-ex/0401025]; V. M. Abazov et al. [D0 Collaboration], Measurement of the $\Lambda_{b}^{0}$ lifetime in the decay $\Lambda_{b}^{0} \rightarrow J / \psi \Lambda^{0}$ with the DØ detector, Phys. Rev. Lett. 94 (2005) 102001 arXiv:hep-ex/0410054; D. E. Acosta et al. [CDF Collaboration], Measurement of $B$ meson lifetimes using fully reconstructed $B$ decays produced in $p \bar{p}$ collisions at $\sqrt{s}=1.8$ TeV, Phys. Rev. D 65 (2002) 092009; K. Abe et al. [BELLE Collaboration], Improved measurement of CP-violation parameters sin(2phi(1)) and 
$\mid$ lambda|, B meson lifetimes, and B0 anti-B0 mixing parameter Delta(m(d)), Phys. Rev. D 71 (2005) 072003 [Erratum-ibid. D 71 (2005) 079903] [arXiv:hep-ex/0408111]; B. Aubert et al. [BABAR Collaboration], Measurement of the $\bar{B}^{0}$ lifetime and the $B^{0} \bar{B}^{0}$ oscillation frequency using partially reconstructed $\bar{B}^{0} \rightarrow D^{*+} \ell^{-} \bar{\nu}_{\ell}$ decays, Phys. Rev. D 73 (2006) 012004 arXiv:hep-ex/0507054.

[25] C. D. Carone and R. Primulando, Constraints on the Lee-Wick Higgs Sector, arXiv:0908.0342 [hep-ph].

[26] C. Amsler et al. [Particle Data Group], Review of particle physics, Phys. Lett. B667 (2008) 1; P. Achard et al. [L3 Collaboration], Search for heavy neutral and charged leptons in $e^{+} e^{-}$ annihilation at LEP, Phys. Lett. B 517 (2001) 75.

[27] A. Strumia and F. Vissani, Neutrino masses and mixings and..., arXiv:hep-ph/0606054. G. Altarelli, Status of Neutrino Masses and Mixing in 2009, arXiv:0905.3265 [hep-ph] and references therein. 